Pacheco, J. (2012), Determinantes socioeconómicos de la educación, la mortalidad $y$ el acceso al agua potable y el saneamiento: un análisis econométrico, PNUD y UN-DESA

Ponce (2012), Determinates del acceso y la permanencia en la educación, la mortalidad de la niñez y materna y el acceso a servicios básicos de agua y saneamiento: Evidencia para el Estado Plurinacional de Bolivia, Quito: FLACSO-Ecuador

Ponce, Juan, Arjun Bedi, and Rob Vos. 2003. “CCómo hacer más eficiente el gasto educativo?” ¿In Quién se beneficia del gasto social en el Ecuador?: desafios para mejorar la calidad y eficiencia del gasto social, by Rob Vos, 89-118. Unidad de Información y Análisis, SIISE de la Secretaría Técnica del Frente Social.

Rosales, Susset. 2006. "Influencia De Variables Socioeconómicas En El Proceso Educativo."

Sabonge, Kenia. (2004) "Factores Asociados a la asistencia escolar en la población de 5 a 19 años. Honduras 2003, Universidad de Costa Rica.

Sánchez, Marco V., and Giacomo Sbrana. 2010. "Determinants of Education Attainment and Development Goals in Yemen". Department of Economic and Social Affairs.

Sánchez, Marco V., and Giacomo Sbrana. 2009. "Determinants of Attendance in Jordan's Education."

Sánchez, Marco V., and Rob Vos. 2010. "Latin America and the Caribbean's Challenge to Reach the MDGs: Financing Options and Trade-offs." DESA Working Paper 74 (May).

SDP, INE, SESAL e ICF (2013), Encuesta Nacional de Demografía y Salud ENDESA 20112012.

Secretaría de Salud. (2002). Análisis de Mortalidad Materna año 2002 Honduras. . Tegucigalpa.

SEFIN. (2012). Cifras Fiscales Mayo de 2012. Tegucigalpa.
Sistema de la Naciones Unidas en Honduras (2010). Objetivos de Desarrollo del Milenio, Honduras 2100

Torres, S., \& Sequeira, S. (2003). Investiogación Cualitativa sobre la Mortalidad Materna en Honduras. Honduras: Instituto Nacional de la Mujer (INAM).

UNICEF. (Diciembre de 2012). Honduras . Recuperado el 30 de Enero de 2013, de Unicef: http://www.unicef.org/honduras/14352_24064. htm

Vos, Rob, Marco V. Sánchez, Enrique Ganuza, Hans Lofgren, and Carolina Díaz-Bonilla, ed. 2010. Public Policies for Human Development: Achieving the Millennium Development Goals in Latin America. New York: Palgrave Macmillan.

Vos, Rob, Marco V. Sánchez, and Cornelia Kaldewei. 2008. "Latin America and the Caribbean's Challenge to Reach the MDGs: Financing Options and Trade-offs." DESA Working Paper 68 (September).

Vos, Rob, J. Cuesta, M. León, R. Lucio y J. Rosero. 2004. "Health". En: World Bank and Inter-American Development Bank (2004) Ecuador: Creating fiscal space for poverty reduction. A fiscal management and public expenditure review, Volume II, Report No. 28911-EC, Washington, D.C.: The World Bank.

Vos, Rob, and Juan Ponce. 2004. "Education." In Ecuador: Creating Fiscal Space for Poverty Reduction, by The World Bank and InterAmerican Development Bank, Volume II:33-66. A Fiscal Management and Public Expenditure Review 28911. The World Bank.

World Health Organization - UNICEF. (2010). Progress in Sanitation and Drinking-Water 2010 .NY: Unicef.

World Health Organization (2003). Global strategy for infant and young child feeding. Geneva: World Health Organization.

World Health Organization (2006). Report of a WHO technical consultation on birth spacing. Geneva: World Health Organization. 
Determinantes de las Metas de

EDUCACIÓN Y LOS OBJETIVOS DE

Desarrollo del Milenio en Honduras

\section{NOTAS}




\title{
RUMBO DE LA EDUCACIÓN SUPERIOR EN HONDURAS, 1995-2015
}

\author{
Manuel Antonio Flores Fonseca \\ Universidad Nacional Autónoma de Honduras (UNAH) \\ Facultad de Ciencias Económicas, Administrativas y Contables (FCEAC) \\ Instituto de Investigaciones Económicas y Sociales (IIES) \\ Ciudad universitaria, edificio C2, primer piso, Tel/fax: + (504) 2216-6100 Ext. 100894 \\ ORCID 0000-0003-4929-7354 \\ manuel.flores@unah.edu.hn
}

DOI http://dx.doi.org/10.5377/eya.v8i2.5616

\section{RESUMEN}

Es un panorama de los rumbos de la educación superior en Honduras, específicamente las tendencias y cambios que se han producido en los últimos veinte años, cuando se producen muchas transformaciones, entre ellas se profundiza la participación del sector privado, la feminización de la matriculación y de los graduados, las preferencias de ciertas áreas de estudio que tienen los demandantes por carreras específicas, considerando estos como algunos de los "rumbos de la educación superior", que responden a factores internos y externos, entre ellos: Los cambios demográficos, el mayor acceso de los jóvenes a la educación terciaria, especialmente de mujeres, las tendencias de empleabilidad del mercado laboral y lógicamente de la globalización.

Palabras clave: Educación Superior, Estadísticas de Educación Superior, 


\title{
COURSE OF HIGHER EDUCATION IN HONDURAS, 1995-2015
}

\author{
Manuel Antonio Flores Fonseca \\ Universidad Nacional Autónoma de Honduras (UNAH) \\ Facultad de Ciencias Económicas, Administrativas y Contables (FCEAC) \\ Instituto de Investigaciones Económicas y Sociales (IIES) \\ Ciudad universitaria, edificio C2, primer piso, Tel/fax: + (504) 2216-6100 Ext. 100894 \\ ORCID 0000-0003-4929-7354 \\ manuel.flores@unah.edu.hn
}

DOI http://dx.doi.org/10.5377/eya.v8i2.5616

\begin{abstract}
It is an overview of the directions of higher education in Honduras, specifically the trends and changes that have occurred in the last twenty years, when there are many transformations, among them the participation of the private sector, the feminization of enrollment and of graduates, the preferences of certain areas of study that the applicants have for specific careers, considering these as some of the "courses of higher education" that respond to internal and external factors, among them, demographic changes, the greater Access for young people to tertiary education, especially for women, trends in the employability of the labor market and, logically, globalization.
\end{abstract}

Keywords: Higher Education, Higher Education Statistics, Higher Education Honduras 


\section{INTRODUCCIÓN}

El número de estudiantes de educación terciaria ha crecido vertiginosamente a nivel mundial en los últimos cuarenta años, de una población estudiantil de alrededor de 30 millones en los años setenta se ha multiplicado más de seis veces hasta alcanzar más de 195 millones en el 2012, aunque hay que apuntar que este crecimiento no ha sido uniforme en el mundo, los países en desarrollo superan desde la década de los ochenta a los países desarrollados (Tabla No.1).

En relación con el acceso de las mujeres en la matriculación de la educación superior hay una tendencia a la feminización, esto se manifiesta en los cambios que ha sufrido el porcentaje de mujeres en la matrícula de ese nivel, en 1970 apenas alcanzaba el $41.3 \%$, recién en la última década supera la mitad, en el 2012 llega al 50.5\%.

Las tasas brutas de la educación superior denotan un incremento general, aunque también muestran las desigualdades regionales, no solo en sus niveles, sino cómo evolucionan a través del tiempo. Es de hacer notar, que las regiones de América del Norte y toda Europa, es decir los países desarrollados, tienen tasas de matrícula que son abismalmente mayores con respecto a las demás regiones mundiales (UNESCO, 2009).

La mayoría de los países no reporta datos por campos de estudio, pero en 80 países se exhiben variaciones en la participación de la educación superior y del tamaño de sus sectores terciarios. Aun así, los datos revelan que las "Ciencias Sociales, Educación Comercial y Derecho" son el primer campo seleccionado por los graduados.

Los estudiantes que seleccionan "Educación" representan un tercio o más del total de graduados en varios países y en muchos otros exhiben un nivel de participación terciaria relativamente bajo. No hay que olvidar que la docencia y otros empleos del sector público constituyen las principales oportunidades laborales para personas coneducación terciaria. Por otra parte, los países que exhiben un nivel de participación terciaria relativamente alto suelen tener una menor proporción de graduados en Educación. El segundo campo más común es
"Ingeniería, Industria y Construcción”, que genera uno de cada diez graduados en más de dos tercios de los países.

América Latina vive actualmente la masificación de la educación superior, ésta ha pasado en catorce años de 7 millones a 18 millones de estudiantes universitarios matriculados (1994-2008), donde los grandes contribuyentes son indiscutiblemente Brasil y en menor volumen México, Argentina, Venezuela, Colombia y Perú.

Según la UNESCO alrededor del año 2006, más de 14,5 millones de estudiantes universitarios estaban matriculados en la región, con una alta concentración en ocho países, e incluso una sola carrera en casi todos los países (administración de empresas) concentraba un tercio del total de la matrícula. La tasa de matriculación promedio en América Latina y la tasa de graduación promedio son bajas, lo que muestra un bajo nivel de eficiencia (Moreno-Brid \& Ruíz-Nápoles, 2009).

En los países de la región hay una tendencia de incremento de la participación de las instituciones de educación superior privadas de forma constante. En la gran mayoría de países, la participación femenina rebasaba ya el $50 \%$ de la matrícula y en algunos países, entre otros del Caribe, representaba más del $60 \%$.

Con este contexto global y regional, se presenta este trabajo de investigación que tiene por objetivo; analizar las tendencias que tienen la educación superior hondureña en las últimas décadas y la influencia que tiene del contexto nacional e internacional. Es de apuntar que este trabajo toma como base los comportamientos estadísticos de la educación superior y de otras variables contextuales.

\section{TEORÍA}

En el mundo de la globalización que se reproduce también en la educación superior implica cambios, transformaciones y desde luego oportunidades y grandes desafíos, principalmente en los países en desarrollo, que viven la expansión cuantitativa, privatización, cambios institucionales $\mathrm{y}$ 
restricciones financieras. Las tendencias son visibles en la masificación, virtualización, privatización, inequidad, evaluación, acreditación, ranking, influencia de las políticas de Estados y del sistema capitalista.

En América Latina las transformaciones que se han producido en los últimos años han llevado a obtener grandes adelantos en la educación superior, aún no han contribuido de manera perceptible a transformaciones progresivas de movilidad social, principalmente en los estratos bajos, que continúan siendo excluidos en el acceso a la educación en los niveles superiores (López Segrera, 2008).

\section{RESULTADOS}

\section{Contexto Nacional de la Educación Superior en Honduras.}

La educación superior hondureña data de 1847 y transcurren 130 años con solamente una institución, no es sino hasta 1978 cuando se abren dos nuevos centros, en la década de los ochenta cuatro centros, en la década de los noventa seis centros y en la última década siete centros para hacer un total de veinte centros actualmente. En este devenir del tiempo, se ha pasado de grandes campus urbanos a multi-campus que, aunque también son urbanos están diseminados en los principales centros urbanos del país.

La oferta educativa de educación superior hasta el 2015 es de 438 grados, de los cuales el 51\% es ofrecido por las instituciones privadas y 49 por las públicas (Honduras, 2015). En la oferta de grados la Licenciatura (52\%), sigue siendo el grado de mayor oferta y aceptación estudiantil para realizar estudios, ya que los superiores fueron ofertados más recientemente.

A través del tiempo ha existido una concentración espacial de la educación superior, primero en la ciudad capital y desde la década del sesenta en las tres principales ciudades del país, no es sino hasta inicios de la década de los ochenta que se expandió a otras ciudades del occidente, sur, centro y oriente del país; primero en la oferta de centros de naturaleza pública y más recientemente de carácter privado, principalmente desde fines de los noventa e inicios del dos mil. Todavía persiste una alta concentración educativa en Tegucigalpa y San Pedro Sula, las dos ciudades más pobladas del país.

\section{Masificación de la Matrícula de la educación superior en Honduras.}

La educación superior de Honduras en la última década alcanzo los niveles más grandes de su historia, en los últimos dieciséis años se incrementó el número de estudiantes matriculados en más de tres veces, actualmente el número de efectivos supera los ciento ochenta y tres mil estudiantes (Figura No.1). Por modalidad la educación presencial es la preponderante, ya que la modalidad virtual ha sido insignificante e incluso contradictoriamente con las tendencias globales casi ha desaparecido del sistema, mientras que la educación a distancia en el periodo estudiado aumentó su número y proporción, sin embargo, no supera un cuarto del total. 


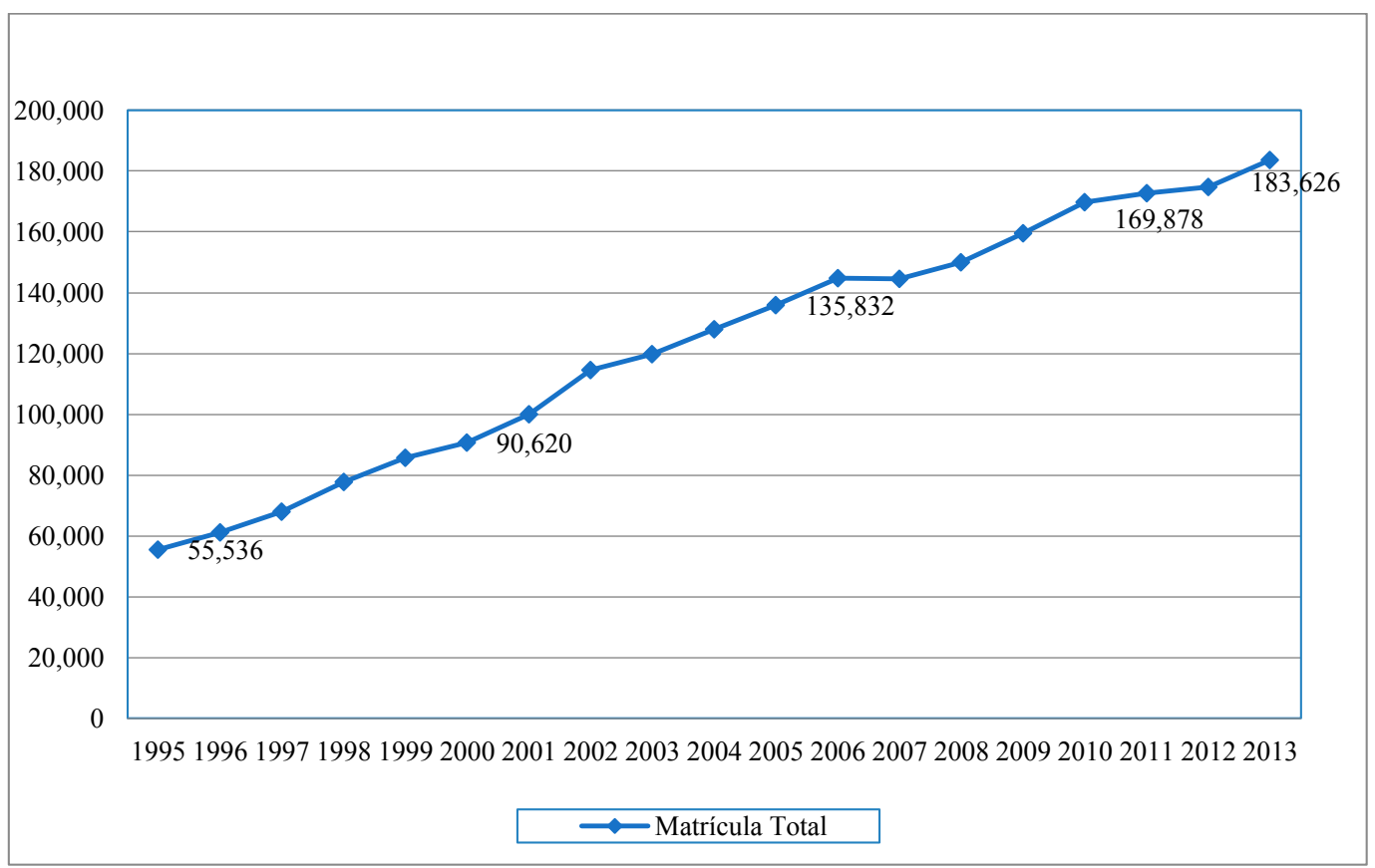

Figura No.1 Honduras: Matrícula Total de Educación Superior, 1995-2013

Fuente: Dirección de Educación Superior de Honduras, 2015

\section{Educación Pública versus Educación Privada en la matriculación Superior.}

En la matricula total de educación superior se ha producido un cambio significativo en el volumen, aunque los centros públicos contribuyen con los mayores volúmenes, en el 2007 disminuyó su número, básicamente por la reducción en la matrícula de la Universidad Nacional Autónoma de Honduras (UNAH), principal contribuyente de la educación superior, que inclusive generó tasas de crecimiento interanual negativas $y$ desde luego, por el incremento de matrícula en los centros privados. En los años de estudio la proporción de matriculados de centros privados se ha incrementado de $13 \%$ a $38 \%$, la cual también es una tendencia en algunos países de la región Latinoamericana.

Por centro público y privado, la UNAH es la mayor contribuyente en estudiantes y supera abismalmente a los demás centros, la Universidad Pedagógica Nacional Francisco Morazán (UPNFM) que también es centro público se convierte en el segundo centro contribuyente de efectivos (Dirección de Educación Superior de Honduras, 2007). En el caso de los demás centros, principalmente los privados contribuyen en volúmenes uniformes, en los últimos la Universidad Tecnológica Centroamericana (UNITEC), la Universidad Católica de Honduras (UNICAH) y la Universidad Tecnológica de Honduras (UTH) han logrado ganar parte del mercado de la matrícula a través de una expansión de su cobertura geográfica, segmentación del mercado de nuevos ingresos y flexibilidad horaria. En suma, estos cinco centros de los veinte existentes absorben más del ochenta y seis por ciento del total de la matrícula, lo que muestra la alta concentración de la educación terciaria.

Concentración de Sectores de Estudio en la matriculación de la educación superior de Honduras.

Los centros superiores por sector de estudio reciben la mayor cantidad de matriculados en las carreras adscritas a las áreas sociales, comerciales y derecho con un promedio cerca del $40 \%$ en los últimos años, en segundo lugar, está 
el sector educativo con un $20 \%$ y en tercer lugar recientemente el sector de la salud y, servicios sociales con un $14 \%$ ha superado al sector de ingenierías, industria y construcción con un promedio de $13 \%$ que han pasado al cuarto lugar (Figura No.2).

En este fenómeno influyen por un lado la oferta educativa de los centros superiores, influenciada por el modelo económico vigente, la oferta de estudios, principalmente la privada que estaba enfocada en el sector de comerciales y derecho, la oferta de estudios a tiempo parcial, principalmente de carácter nocturno, que puede ser complementada con trabajo diurno, sectores del conocimiento donde la oferta educativa da mayores facilidades de horarios de fines de semana, menores exigencias de aprobación de asignaturas y por supuesto la preferencia de los padres y estudiantes.

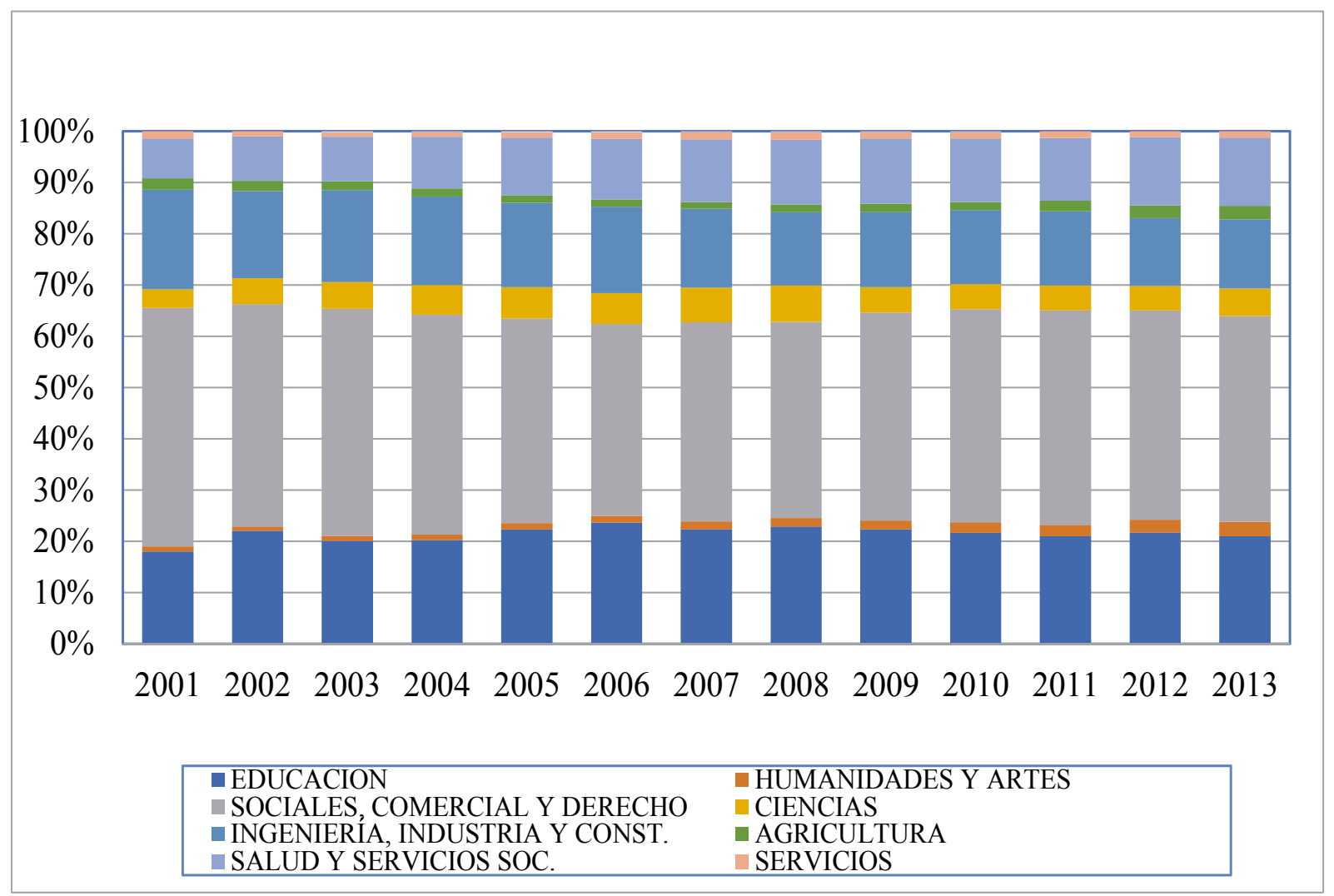

Figura No.2 Honduras: Matrícula Total de Educación Superior por Sectores de Estudio, 2001-2013 (\%) No 10

En los últimos años la oferta privada incursionó en varios sectores que anteriormente eran bastiones de formación pública, entre ellos: la salud y servicios sociales, lo que ha llevado a la diversificación en las opciones ofertadas y a la masificación de estudiantes en esas áreas que exigen menores requisitos de ingreso, aunque los costos de aranceles son elevados.

En general, se observa que la oferta educativa no corresponde a ninguna articulación con la demanda de profesionales, sino más bien a la demanda creciente de nuevos ingresos en edad de la educación superior que aspiran culminar sus pensum en el menor tiempo posible para insertarse en el mundo del trabajo. Esta tendencia de concentración en algunos sectores del conocimiento, también se produce en otros países de América Latina, de manera similar y realmente las mayores ofertas laborales son en esas áreas. 


\section{Feminización en la matriculación de la Educación Superior en Honduras.}

En la matrícula total de los centros de educación superior hondureños prima la feminización de la educación, el índice de masculinidad (IM) en la matricula alcanza el $67 \%$ ?, que significa el alto grado de participación femenina en el mercado estudiantil superior, tendencia regional. En más del $60 \%$ de las instituciones superiores hay más mujeres que hombres matriculadas, principalmente en las dos más grandes instituciones públicas.

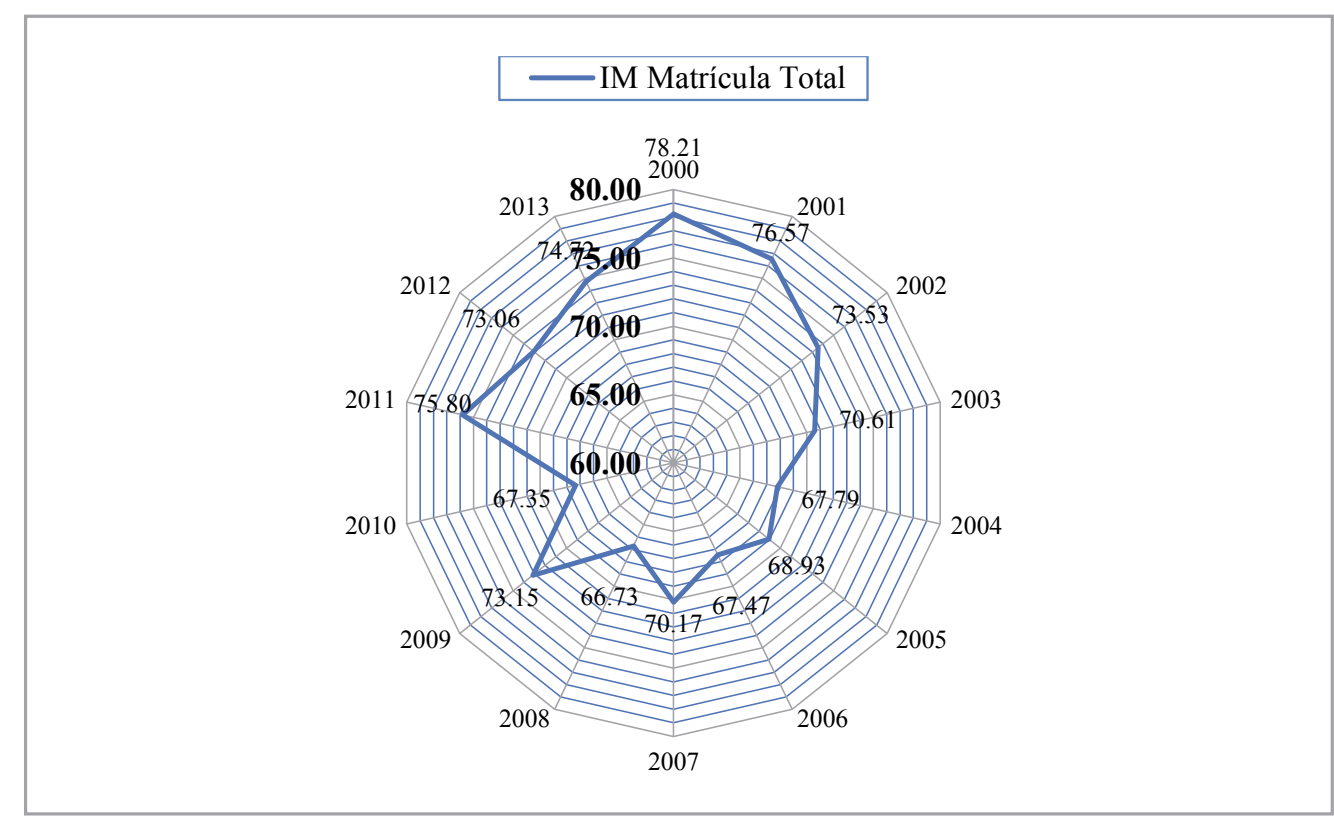

Figura No.3 Honduras: Índice de Masculinidad de la Matrícula Total de la Educación Superior, 2000-2013

Fuente: Dirección de Educación Superior de Honduras, 2015)

\section{Matrícula de Primer Ingreso en la educación superior en Honduras (nuevas entradas)}

Los centros de educación superior han aumentado el número de nuevos ingresos a través del tiempo, en una década (1998-2008) su número aumentó de 17,646 a 27,933 estudiantes, aunque no fue de manera uniforme, ya que en el año 20022003 y el año 2007 se produjeron retrocesos en la tendencia, y se manifestaron en tasas de crecimiento interanual negativas, que en el último año superaron el veinte por ciento.

Esas dos reducciones en la tendencia en el flujo de efectivos de primer ingreso se debieron posiblemente por los picos producidos en la afluencia masiva en la matrícula de los años 20012002 en la UPNFM por los programas de formación docente, que incentivados por mejoras salariales en el estatuto del docente atrajeron ese flujo, que consecuentemente terminada su afluencia en la matrícula de primer ingreso decayó. A su vez, en el año 2006 se introduce en la UNAH un examen de admisión para estudiantes de primer ingreso del primer período del 2007 que provocó un cisma en la afluencia en la mayor universidad del país, que llegó a producir una tasa de crecimiento negativa del sesenta por ciento.

Esa reducción de los nuevos ingresos en la UNAH también se manifestó en la educación superior pública del país, que en los últimos diez años reduce su proporción del total, mientras en 1998 la matrícula de primer ingreso representaba el $81 \%$, ya desde el 2007 se producen desequilibrios, que hacen que algunos años la matrícula de primer ingreso es más alta en los centros privados y otros años en los públicos.

En los nuevos ingresos estudiantiles sobresalen 
las mujeres, en el 2008 el 56\% pertenecen al sexo femenino, e incluso instituciones como la UPNFM y la UNAH sus matrículas de primer ingreso son mayoritariamente femeninas, tendencia que viene de años atrás.

\section{Graduados de Educación Superior en Honduras (Salidas).}

El número de salidas formales de la educación superior en el país se ha incrementado a través del tiempo. En los últimos diez años los graduados pasaron de menos de cinco mil a cerca de veinte mil personas, aunque los años 2001 y 2005 se produjeron cambios en la tendencia incremental, producidas posiblemente por los programas especiales de capacitación docente de la UPNFM en la que graduaron estudiantes en los años anteriores a los efectos de disminución.

Desde hace muchos años se produce la feminización en la educación superior, en el caso de los graduados en la última década es mayoritaria la cantidad de graduadas, incluso en los últimos años más que duplica su cantidad. En los centros de educación superior de mayor contribución en la formación de recursos humanos casi sin excepción se repite la tendencia de feminización de graduados.

\section{Resultados de las Entradas, procesos y salidas en la educación superior en Honduras.}

Un resumen de la población universitaria hondureña muestra que la matrícula total del sistema tiene una tendencia incremental a través del tiempo, lo mismo pasa con la cantidad de graduados cada año, sin embargo, apenas ha superado un décimo de la matrícula, esto indica que la inmensa mayoría se encuentra cursando estudios.

Teóricamente cada cohorte estaría estudiando cinco años en los centros superiores por lo que podría esperarse de acuerdo con el flujo de la matrícula de primer ingreso, que el número de estudiantes matriculados debería ser menor, lo que indica que las cohortes de los estudiantes en las universidades superan los años que teóricamente deben de estar en el sistema, incluso su número se incrementa cada año.
La cobertura educativa de la UNAH, principal centro educativo del país ha tenido un comportamiento contradictorio, primero tuvo incrementos hasta el año 2004 y de allí en adelante ha disminuido su tasa de cobertura, similar situación se presenta en la educación superior pública a excepción que el punto de pico se produce en el año 2006. La cobertura de la educación privada tiene una tendencia alcista a través del tiempo, representando en los últimos años entre $38-40 \%$ de la educación superior.

\section{CONCLUSIONES}

La educación superior hondureña sigue en aumento en su matriculación, aunque todavía la educación terciaria pública es mayoría hay una tendencia relativa a la disminución. Incluso en las matrículas de nuevos ingresos en algunos años la educación privada ha superado a la pública como un hito histórico, en los últimos años la educación privada absorbe la mitad de nuevos ingresos en las universidades hondureñas, lo cual indica el grado de incorporación privada en los niveles superiores de la educación.

En las áreas del conocimiento de Honduras, tiene una alta concentración de la oferta educativa en el sector de ciencias sociales, ciencias comerciales y derecho, que sumadas a la enseñanza de la educación y humanidades resulta elevada alcanzando casi los dos tercios, patrón que sigue las tendencias de concentración en la región e incluso en muchas zonas a nivel mundial.

La feminización de la educación superior es inobjetable tanto a nivel de matriculación como de graduados, esto debido a múltiples factores como el flujo de entradas de mujeres procedentes de la educación media, mayores oportunidades de estudio para las mujeres que conceden las familias en relación con el pasado, mayor dedicación y por ende mejores resultados académicos, en resumen, espacios académicos ganados a través del tiempo.

La cobertura educativa superior en la población del país es todavía baja y sus avances son muy 
lentos, aun cuando las tendencias demográficas apuntan a un incremento en las poblaciones en edad de educación superior y también de los graduados de educación media insumos de la educación terciaria.

En general, los rumbos de la educación superior hondureña transitan en tendencia hacia la masificación, la mayor participación de la educación privada, la feminización y la concentración de los sectores de estudio y que son reflejo del contexto interno y su ligazón con el externo, entre ellos los cambios demográficos, el mayor acceso de los jóvenes a conseguir ingresar a la educación terciaria, especialmente de las jóvenes mujeres, las tendencias de empleabilidad del mercado laboral que responden a la lógica del mercado en su inserción de procesos económicos globales. 


\section{BIBLIOGRAFÍA}

Dirección de Educación Superior de Honduras, 2007. Anuario Estadístico del Nivel de Educación Superior, Tegucigalpa: DES.

Honduras, D. d. E. S. d., 2015. Dirección de Educación Superior - DES. [En línea]

Available at: https://des.unah.edu.hn/servicio-al-usuario/estadisticas/

[Último acceso: 3 de Octubre Octubre 2016].

López Segrera, F., 2008. Tendencias de la Educación
Superioren el Mundo, América Latina y El Caribe. Avaliação, 13(2), pp. 267-288.

Moreno-Brid, J. C. \& Ruíz-Nápoles, P., 2009. La Educación Superior y el Desarrollo Económico en América Latina, México: CEPAL.

UNESCO, 2009. Compendio Mundial de la Educación 2009, Comparación de las estadísticas de educación en el mundo, Montreal: Instituto de Estadística, UNESCO. 
Tabla No.1: Matrícula Total de la Educación Superior según Clasificaciones Regionales, 1970-2012

\begin{tabular}{|c|c|c|c|c|c|}
\hline \multirow[b]{2}{*}{ Educación para Todos } & 1970 & 1980 & 1990 & 2000 & 2012 \\
\hline & & & & & \\
\hline Mundial & 32433,983 & 49538,399 & 67222,986 & 99180,321 & 195556,567 \\
\hline Países Desarrollados & 17075,536 & 24245,952 & 29484,647 & 35844,833 & 48567,283 \\
\hline Países en Desarrollo & 6885,167 & 15460,922 & 28388,913 & 52785,867 & 133255,137 \\
\hline Países en Transición & 8473,279 & 9831,525 & 9349,426 & 10549,621 & 13734,147 \\
\hline Países Árabes & & 1587,055 & 2391,249 & 5037,722 & 8888,273 \\
\hline Europa Central y Oriental & 8759,253 & 10040,923 & 9835,729 & 14024,391 & 20778,873 \\
\hline Asia Central & & 1477,958 & 1540,232 & 1476,927 & 2077,185 \\
\hline Asia Oriental y Pacifico & 3577,030 & 7038,470 & 13768,320 & 24968,505 & 58196,437 \\
\hline América Latina y El Caribe & 1573,587 & 4666,160 & 7016,204 & 11192,276 & 22890,625 \\
\hline $\begin{array}{l}\text { América del Norte y Europa } \\
\text { Occidental }\end{array}$ & 14060,873 & 20152,770 & 25031,681 & 27770,280 & 38860,500 \\
\hline Asia Meridional y Occidental & 2676,659 & 4001,388 & 6337,457 & 12163,899 & 37522,426 \\
\hline Africa Subsahariana & 213,059 & 573,676 & 1302,112 & 2546,320 & 6342,249 \\
\hline $\begin{array}{l}\text { Regiones Educación Global } \\
\text { Digest }\end{array}$ & & & & & \\
\hline Mundial & 32685,930 & 49734,302 & 67543,134 & 99527,915 & 196077,086 \\
\hline Países Árabes &... & 1587,055 & 2391,249 & 5037,722 & \begin{tabular}{|l}
8888,273 \\
\end{tabular} \\
\hline Europa Central y Oriental & 8759,253 & 10040,923 & 9835,729 & 14024,391 & 20778,873 \\
\hline Asia Central & & 1477,958 & 1540,232 & 1476,927 & 2077,185 \\
\hline Asia Oriental y Pacifico & 3600,821 & 7095,241 & 13849,313 & 25092,234 & 58466,275 \\
\hline América Latina y El Caribe & 1801,302 & 4795,868 & 7243,920 & 11415,701 & 23140,321 \\
\hline $\begin{array}{l}\text { América del Norte y Europa } \\
\text { Occidental }\end{array}$ & 14061,313 & 20162,194 & 25043,121 & 27770,720 & 38861,484 \\
\hline Asia Meridional y Occidental & 2676,659 & 4001,388 & 6337,457 & 12163,899 & 37522,426 \\
\hline Africa Subsahariana & 213,059 & 573,676 & 1302,112 & 2546,320 & 6342,249 \\
\hline $\begin{array}{l}\text { Regiones de las Metas del } \\
\text { Milenio }\end{array}$ & & & & & \\
\hline Mundial & 32685,930 & 49725,318 & 67532,134 & 99527,915 & 196077,086 \\
\hline Países Desarrollados & 24749,247 & 32738,195 & 37462,738 & 45258,297 & 60796,522 \\
\hline Países en Desarrollo & 7936,683 & 16987,123 & 30069,396 & 54269,618 & 135280,564 \\
\hline Caucaso y Asia Central & & 1438,868 & 1500,194 & 1402,902 & 1903,329 \\
\hline América Latina y El Caribe & 1800,934 & 4795,301 & 7243,146 & 11414,769 & 23139,043 \\
\hline Africa del Norte & 352,745 & 958,426 & 1359,283 & 3323,687 & 4734,228 \\
\hline Africa Subsahariana & 385,668 & 747,023 & 1480,191 & 2738,166 & 6916,030 \\
\hline Asia Occidental & 333,429 & 735,061 & 1539,387 & 3059,098 & 7933,806 \\
\hline Asia Oriental & 419,209 & 1823,655 & 6004,447 & 10934,345 & 37168,677 \\
\hline Asia Sur Oriental & 1107,705 & 2476,915 & 4585,156 & 9205,516 & 15931,658 \\
\hline Asia Meridional & 2676,659 & 4001,388 & 6337,457 & 12163,899 & 37522,426 \\
\hline Oceanía &.. & 10,487 & 20,134 & 27,235 & \\
\hline Regiones UNESCO &.. &.. &.. &.. & .. \\
\hline Mundial & 32685,930 & 49734,302 & 67543,134 & 99527,915 & 196077,086 \\
\hline Africa & 738,412 & 1705,449 & 2839,474 & 6061,853 & 11650,258 \\
\hline Asia & 7310,735 & 12997,947 & 22778,097 & 41014,134 & 104741,971 \\
\hline Europa & 13416,813 & 16869,891 & 18686,093 & 25576,856 & 32524,016 \\
\hline América del Norte & 9792,377 & 14430,120 & 17694,936 & 17680,587 & 27814,783 \\
\hline América del Sur & 1201,095 & 3320,049 & 4927,821 & 8150,155 & 17690,901 \\
\hline Oceanía & 226,498 & 410,846 & 616,713 & 1044,329 & 1655,158 \\
\hline $\begin{array}{l}\text { Regiones Agrupación de } \\
\text { Ingresos Banco Mundial }\end{array}$ & & &.. & & \\
\hline Países de bajos ingresos & 366,615 & 862,360 & 1550,319 & 2468,266 & 7182,883 \\
\hline $\begin{array}{l}\text { Países de ingresos medios } \\
\text { bajos }\end{array}$ & 5974,308 & 9166,406 & 13215,422 & 23759,048 & 53697,039 \\
\hline Países de ingresos medios & 9636,036 & 18113,620 & 28984,338 & 51053,190 & 126252,999 \\
\hline Países de renta media-alta & 3661,728 & 8947,213 & 15768,915 & 27294,142 & 72555,960 \\
\hline Países de altos ingresos & 22682,423 & 30748,482 & 36996,621 & 46005,708 & 62639,328 \\
\hline
\end{tabular}

(Fuente: UIS-UNESCO, 2015) 


\section{NOTAS}




\title{
EL PROGRAMA DE TRANSFERENCIA MONETARIA DE HONDURAS "BONO 10 MIL": SU CARACTERIZACIÓN E IMPACTO SOCIOECONÓMICO
}

\author{
Margarita Elizabeth Díaz \\ Universidad Nacional Autónoma de Honduras (UNAH) \\ Facultad de Ciencias Económicas, Administrativas y Contables (FCEAC) \\ Instituto de Investigaciones Económicas y Sociales (IIES-UNAH) \\ Ciudad universitaria, edificio C2, primer piso, Tel/fax: + (504) 2216-6100 Ext. 100894 \\ margarita.diaz@unah.edu.hn
}

DOI: $\underline{\text { http://dx.doi.org/10.5377/eya.v8i2.5617 }}$

\section{RESUMEN}

Las transferencias monetarias condicionadas en Honduras son estrategias para llegar a los hogares más postergados y con mayores limitaciones de sobrevivencia, un modelo para aminorar el efecto de las medidas estructurales económicas y que limitaron el acceso al mercado laboral de muchos hondureños desde los años 90's, Lo que produjo un efecto negativo en el mejoramiento de los niveles de pobreza. En este análisis se presentaron una serie de variables socioeconómicas que definen el programa Bono 10 mil, en el periodo 2012-2014 de manera de caracterizarlo y a partir del cual establecer el impacto que ha tenido sobre los hondureños beneficiados por el programa.

Palabras clave: Transferencias Monetarias Condicionadas, Bono 10 mil, Pobreza. 


\title{
THE MONETARY TRANSFER PROGRAM OF HONDURAS "BONO 10 MIL": ITS CHARACTERIZATION AND SOCIOECONOMIC IMPACT
}

\author{
Margarita Elizabeth Díaz \\ Universidad Nacional Autónoma de Honduras (UNAH) \\ Facultad de Ciencias Económicas, Administrativas y Contables (FCEAC) \\ Instituto de Investigaciones Económicas y Sociales s (IIES-UNAH) \\ Ciudad universitaria, edificio C2, primer piso, Tel/fax: + (504) 2216-6100 Ext. 100894 \\ margarita.diaz@unah.edu.hn
}

DOI: $\underline{\text { http://dx.doi.org/10.5377/eya.v8i2.5617 }}$

\begin{abstract}
Conditional monetary transfers in Honduras are a strategy to reach the most deferred households and with greater limitations of survival, a model to alleviate the effect of economic structural measures and that limited the access to the labor market of many Hondurans since the 1990's; which had a negative effect on the improvement of poverty levels. In this analysis, a series of socio-economic variables are shown which define in a "Bono 10 Thousand program", in order to characterize it and from which to establish the impact it has had on the Hondurans who were benefited by the program.
\end{abstract}

Key words: Conditional Monetary Transfers, 10 Thousand Bonus, Poverty. 


\section{I.- INTRODUCCIÓN}

Durante la década pasada Honduras tuvo un comportamiento económico fluctuante, en los años intermedios, de la misma se dio un crecimiento económico alrededor de $5.4 \%$, pero luego tuvo una deceleración del $2.1 \%$ a finales de la década 2001-2010; unido a este panorama, otros indicadores también desmejoraron en su conjunto e impactaron directamente en la renta de los ingresos de los hogares hondureños. En el 2012, el país tenía ingresos relativamente bajos, con un ingreso nacional bruto per cápita de US\$4,270.00, ubicando a Honduras en el puesto 162 a nivel mundial.

Por otro lado, hay que reconocer que se han hecho esfuerzos por disminuir la pobreza, para mejorar las condiciones de vida de la población, mediante el incremento o el acceso a la salud y los niveles educativos de los niños y jóvenes; sin embargo, Honduras sigue siendo uno de los países más pobres de la región, con muchas desigualdades e iniquidades. Aún faltan esfuerzos para revertir esta situación, siguen siendo insuficientes los programas y proyectos en las áreas de salud, educación, nutrición y empleo.

En este contexto, se han implementado proyectos para subsanar el impacto de las condiciones socioeconómicas y para mejorar las condiciones de pobreza; básicamente se han orientado programas y proyectos para atender necesidades de educación, salud y nutrición de los hogares que se encuentran en pobreza y pobreza extrema. Estos proyectos están diseñados dentro de los programas denominados "Programas de Transferencias Condicionadas", $o$ aquellos incentivos en efectivo que se da a familias que cumplan con requisitos y conductas como: i.- Enviar sus hijos menores a la escuela, ii.- Llevar a éstos a chequeos médicos, lo que en general aumentan la escolaridad de los niños, mejora la cobertura de atención en salud e incrementa los niveles de consumo de los más pobres, con una consecuente mejoramiento de la calidad de vida de estos.
El análisis del programa monetario condicionado está basado en las variables i.- Dominio urbanorural, ii.- Nivel educativo del jefe de hogar, iii.Condición de actividad económica del jefe de hogar, iv.- Clasificación de pobreza, v.- Ingreso mensual de hogar, vi. - Sexo del jefe de hogar.

\section{II.- OBJETIVOS}

i.- Describir el contexto general del Programa de Transferencia Monetaria Condicionada "Bono 10 mil"

ii.- Evaluar el programa de transferencia condicionada (Bono 10k).

iii.- Definir la cobertura y su vinculación con la población objetivo.

\section{III.- DESCRIPCIÓN DE LAMETODOLOGÍA}

Etapas: Se realizaron las siguientes tareas para procesar los indicadores y establecer las tendencias respectivas: i.- Priorización de enfoque lógico, ii.- Identificación de grupos de población beneficiaria de las transferencias condicionadas, ii.- Procesamiento de indicadores socioeconómicos y su vinculación con el Bono 10 mil, y iv.- La integración de los resultados obtenidos permitirá identificar los escenarios posibles.

Enfoque: El enfoque descriptivo y responde más a una caracterización en base a los datos disponibles de varias fuentes, lo que permitió no solo la comparación, sino la escogencia de indicadores que fueran más robustos y coincidentes con las fuentes nacionales. Dicha caracterización permite dar a conocer lo existente, sin esperar calcular indicadores que corren el riesgo de sub-registro o ausencia de ellos, sin embargo, da suficientes indicios para el análisis del tema y para darle una continuidad a futuro.

Las fuentes de datos: Se tomaron en cuenta 
las estadísticas oficiales del Instituto Nacional de Estadísticas (INE) y otras fuentes de reconocida credibilidad. Se utilizaron los datos de las Encuestas de Hogares de Propósito Múltiples del 2012 al 2014, para establecer la tendencia de datos de pobreza y empleo, se recurrió a los censos de población del 2001 y 2013, adicionalmente, se contrastó otras fuentes externas de organismos especializados, para verificar las cifras nacionales y en muchos casos para llenar los espacios de información no encontrados.

Unidad de análisis: Son grupos de edades de la población vinculados a la protección social. Los grupos inactivos, grupo de menores de 14 años y el grupo de 60 años y más; y como grupo activo, el grupo de 15-59 años.

Variables: 1.- Dominio: urbano, rural, 2.Grupos funcionales de edad. Menor de 14 años, entre 15-59 años, de 60 y más años, 3.Por departamento, 4.- Condición de actividad (para ubicar los desempleados), 5.- Nivel educativo, 6.- Clasificación de pobreza, 7.Ingreso mensual, 8.- Sexo del jefe de hogar $\mathrm{X}$ Dominio, 9.- Quintil, 10.-Transferencias o bonos recibidos. Variables que se tomaron de la Encuesta de Hogares de Propósitos Múltiples del 2012-2014: Pregunta clave: DH10: ¿Este hogar está siendo beneficiado con el programa del bono 10 mil?

\section{MARCO CONTEXTUAL}

\section{Contexto de la Protección Social}

\section{Programas de Transferencias Monetarias Condicionadas}

Los programas de transferencias monetarias condicionadas puntualmente son los programas de la protección social, que buscan por medio de la transferencia de donaciones en efectivo estimular el desarrollo de la población a través de un conjunto de corresponsabilidades o condicionalidades, que se centran principalmente en la salud y educación infantil, y en algunos casos también en la salud materna.

Los programas de protección social fueron implementados por varias razones: i.- La economía del país se estancó, lo que generó altas tasas de pobreza y de trabajo informal. Grandes grupos de población carecieran de la cobertura de planes de seguridad social asociados al empleo formal (Fonseca, 2006). ii.- Promoción del concepto de "crecimiento pro-pobre", que incluía oportunidades de empleo y políticas públicas dirigidas a mitigar las desigualdades, con especial énfasis en las mujeres y en grupos tradicionalmente excluidos. Se incorporó la idea de que las inversiones en necesidades humanas básicas mejoran la productividad y contribuyen a la eficiencia y al crecimiento económico, según (Kakwani y Pemia, 20009); (Eastwood y Lipton, 2001); (Ravallion, 2004) citado por BID, 2013.

iii.- La reactivación del crecimiento económico amplió el espacio fiscal para asistencia social en la década del 2000 (Ver Tabla No.1).

Tabla No. 1: Crecimiento económico de Honduras

\begin{tabular}{|l|l|l|l|l|}
\hline País & \multicolumn{4}{|c|}{ Período } \\
\hline & $\mathbf{1 9 8 0 - 2 0 0 2}$ & $\mathbf{2 0 0 3 - 2 0 0 8}$ & $\mathbf{2 0 0 9}$ & $\mathbf{2 0 1 0 - 2 0 1 1}$ \\
\hline Honduras & 2.9 & 5.4 & -2.1 & 3.2 \\
\hline
\end{tabular}

Fuente: Indicadores del Desarrollo Mundial (consultados el 11 de enero de 2013). 
iv.- Las transferencias monetarias condicionadas fueran vistas como una forma de redistribución con estándares morales y técnicos aceptables. La economía política de los programas contribuyó a garantizar el apoyo de políticos y votantes, que valoraron la transparencia de los procesos de focalización de beneficiaros de los programas de transferencias monetarias condicionadas y la exigencia a los beneficiarios de cumplir con su parte de la responsabilidad, haciendo de la participación un contrato implícito con obligaciones tanto para los gobiernos como para los hogares pobres según (Fiszbein y Schady, 2009) citado por BID, 2013.

Dentro de muchos proyectos sociales que se han estado ejecutado, se observan 2 programas prioritarios de protección social: i.- El programa Bono 10 mil y ii.- El programa Alimento Solidario para el adulto mayor (ASAM), y un tercer programa propuesto más recientemente, el Programa de Apoyo al Bono Vida Mejor implementado por el actual gobierno para reforzar la Política de Protección Social. En la Tabla No. 2 puede apreciarse dichos programas. Sin embargo en el contexto de esta tesis se enfocará en el Programa Bono 10 mil, por ser un programa piloto alrededor del cual se han ido modificándose otros programas y por haberse monitoreado y evaluado completamente en su ejecución en el cual puede verse una evolución completa

Tabla No. 2 Programas de protección social en Honduras

\begin{tabular}{|c|c|c|c|c|}
\hline Programas & Beneficiarios & Condicionalidades & Beneficios & Financiador / Presupuesto \\
\hline $\begin{array}{l}\text { Programa Bono } \\
10 \text { Mil urbano }\end{array}$ & $\begin{array}{l}350 \text { mil hogares po- } \\
\text { bres, } 250.000 \text { rurales y } \\
100.000 \text { urbanos } \\
1.5 \text { millones de per- } \\
\text { sonas }\end{array}$ & $\begin{array}{l}\text { Familias en condiciones en ex- } \\
\text { trema pobreza con menores de } 14 \\
\text { años que asistan a la escuela, y a } \\
\text { controles de los servicios de salud }\end{array}$ & $\begin{array}{l}\text { Transferencia en efectivo } \\
\text { por familia de L. } 10,000.00 \\
\text { anual/ } \\
\$ 45.00 \text { mensual }\end{array}$ & $\begin{array}{l}* \text { BM } \\
\$ 40 \text { Millones } \\
* * \text { AIF } \\
\$ 12.3 \text { Millones } \\
* * * \text { BID } \\
\text {-\$100 millones } \\
\text { - US \$55 millones (Segunda operación } \\
\text { para el Programa de Apoyo Integral a } \\
\text { la Red de } \\
\text { Protección Social) } \\
\text {-\$75 millones (Programa de Apoyo a } \\
\text { la Red de Protección Social) } \\
\text { Banco Mundial: } \\
\text { 2096/BL-HO, 100\% }(\$ 20 \mathrm{~m}) \\
\text { 2372/BL-HO, 100\% }(\$ 55 \mathrm{~m}) \\
\text { 2737/BL-HO, } 90 \%(\$ 75 \mathrm{~m}) \\
\text { 2937/BL-HO, 25\% }(\$ 100 \mathrm{~m})\end{array}$ \\
\hline $\begin{array}{l}\text { Programa Ali- } \\
\text { mento Solidario } \\
\text { para el adulto } \\
\text { mayor (ASAM) }\end{array}$ & $\begin{array}{l}175,000 \text { beneficiados a } \\
\text { nivel Nacional: } \\
\text { Francisco Morazán: } \\
50,000 \text { Beneficiarios } \\
\text { Cortés: } 40,000 \text { Benefi- } \\
\text { ciarios } \\
\text { Otros departamentos: } \\
85,000 \text { Beneficiarios }\end{array}$ & $\begin{array}{l}\text { Personas mayores de } 65 \text { con dis- } \\
\text { capacidad y de } 70 \text { que están en } \\
\text { pobreza o extrema pobreza, y que } \\
\text { no participen del bono } 10 \text { Mil }\end{array}$ & $\begin{array}{l}\text { Provisión de canasta básica } \\
\text { de alimentos directa a los } \\
\text { beneficiarios mensual }\end{array}$ & Información no disponible \\
\hline $\begin{array}{l}\text { Programa de } \\
\text { Apoyo al Bono } \\
\text { Vida Mejor y a la } \\
\text { Estrategia } \\
\text { Vida Mejor } \\
\text { (antes Bono } \\
10,000)\end{array}$ & $\begin{array}{l}\text { Al menos } 80.000 \text { ho- } \\
\text { gares en zonas rurales } \\
\text { de los departamentos } \\
\text { de Occidente del país }\end{array}$ & $\begin{array}{l}\text { Los hogares beneficiarios firman } \\
\text { un Acta de Compromiso que de- } \\
\text { talla los compromisos en términos } \\
\text { de asistencia escolar y uso de ser- } \\
\text { vicios de salud } \\
\text { 1.-Garantizar de ingreso mínimo a } \\
\text { las familias más pobres, } \\
\text { 2.-Seguridad alimentaria } \\
\text { 3.- Vivienda saludable } \\
\text { 4.-Acceso a educación pre básica } \\
\text { y básica (hasta noveno grado) y } \\
\text { 5.-Atención primaria en salud }\end{array}$ & $\begin{array}{l}\text { La transferencia anual pro- } \\
\text { medio en zonas rurales será } \\
\text { de L. } 7.250 \text { Lempiras por } \\
\text { hogar. Que corresponde al } \\
21 \% \text { del ingreso per cápita } \\
\text { de hogares en pobreza ex- } \\
\text { trema en zonas rurales }\end{array}$ & $\begin{array}{l}\text { Del } 1 \text { junio de } 2015 \text { al } \\
31 \text { diciembre } 2018 \\
\text { BID: } \\
\text { US\$76.909.000 (CO) US\$32.961. } \\
\text { (FOE)) US } \$ 10.987 .000 \text { de aporte local }\end{array}$ \\
\hline
\end{tabular}

Fuente: Elaboración propia en base a datos de la Secretaría de Estado de Desarrollo e Inclusión Social

*Banco Mundial, **Agencia Internacional de Fomento, ***Banco Interamericano de Desarrollo 


\section{Programa de Transferencias Monetarias Condicionadas en Honduras Bono $10 \mathrm{Mil}$}

El programa de transferencias monetarias condicionadas, denominado "Programa Presidencial de Salud, Educación y Nutrición, o "Bono 10.000", ha sido un pilar clave en la estrategia de desarrollo social de Honduras. Asimismo, este programa ha contribuido de forma positiva con los lineamientos estratégicos con los cuales se pretende contribuir con los diferentes ejes de intervención de la Estrategia de Reducción de la Pobreza (ERP): i.- Protección; ii.- Capacidades; iii.- Infraestructura social y productiva; y iv.-Oportunidades de ingreso como una estrategia de país.

Como una estrategia de país, el programa de transferencias monetarias condicionadas, el Programa Bono 10,000, ha sido un pilar clave en la estrategia de desarrollo social de Honduras. Asimismo, este programa contribuyó de forma positiva con los lineamientos estratégicos por medio de los cuales se pretende contribuir con los diferentes ejes de intervención de la Estrategia de Reducción de la Pobreza (ERP): i.- protección; ii capacidades; iii.- infraestructura social y productiva; y iv.- oportunidades de ingreso.

\section{Objetivos de desarrollo}

El objetivo general del programa: Reducir la transmisión inter-generacional de la pobreza mediante la consolidación del Programa Presidencial de Salud, Educación y Nutrición "Bono 10,000".

Los objetivos específicos: (i) Apoyar la expansión de la cobertura del Programa Bono 10,000 y su consolidación; (ii) Fortalecer la SDP en su rol rector y coordinador del Programa Bono 10,000 así como la Coordinación Nacional del Bono 10,000 y al PRAF; y (iii) Mejorar la eficiencia en la entrega y monitoreo de las transferencias monetarias condicionadas. El programa estuvo compuesto por dos componentes: (i) Componente 1: Transferencias monetarias con corresponsabilidades; y (ii) Componente 2: Fortalecimiento Institucional.
Caracterización de los beneficiarios Bono 10,000.

De acuerdo con los informes de monitoreo y evaluación del programa, se cubrió aproximadamente al $40 \%$ de los hogares pobres extremos y $38 \%$ de los hogares pobres en zonas rurales (11\% y $9 \%$ en zonas urbanas). Las familias beneficiarias tienen en promedio de 3,2 hijos entre 0 y 18 años, la tasa de asistencia en educación básica $\left(1^{\circ}\right.$ a $\left.6^{\circ}\right)$ es de $91,6 \%$ y en el 3 er ciclo es de $52 \%$; el promedio de años de educación formal del jefe de hogar es menor a siete años de educación. En cuanto a salud, los indicadores de los beneficiarios están muy por debajo de los niveles nacionales, 33,6\% cuenta con un calendario de vacunación completo comparado con el $74,9 \%$, y la prevalencia de anemia para niños de 6 a 23 meses es de $68 \%$ comparado con $56,5 \%$.

\section{Financiamiento}

En importante señalar que Banco Mundial ha aportado US\$ 40 millones en su etapa inicial. Dicho programa comenzó en el 2010 por medio de un préstamo, con un presupuesto inicial de US\$ 40.00 millones, con un período de gracia de los primeros 5 años y pagaderos a 15 años, lo que implica un compromiso desde ya del Estado para asumir dicho préstamo.

El BID por otro lado, ha prestado US $\$ 100$ millones, de los cuales US\$70 millones son del capital ordinario, con un plazo de 30 años, en un período de gracia de 5,5 años y una tasa de interés basada en LIBOR, y US\$30 millones del Fondo para Operaciones Especiales, por un plazo de 40 años y un período de gracia de 40 años y 0,25 por ciento de interés. La contraparte local es de US\$10 millones.

El financiamiento adicional de $\$ 12.3$ millones de dólares para el Proyecto contempla \$9.89 millones de dólares para financiar transferencias monetarias para hogares del programa Bono $10 \mathrm{Mil}$ y \$2.41 millones de dólares para fortalecimiento institucional. 
El Directorio Ejecutivo del Banco Mundial aprobó la extensión y el financiamiento adicional del Proyecto de Protección Social el 8 de agosto de 2013, por lo que su implementación se extenderá hasta el 31 de diciembre de 2015. El crédito de $\$ 12.3$ millones de dólares fue proporcionado por la Agencia Internacional de Fomento (AIF) a un plazo de 25 años, incluido un período de gracia de 5 años.

\section{RESULTADOS DEL ESTUDIO}

\section{Resultado de la post evaluación de impacto del Bono 10000}

En el 2013 se llevó a cabo una evaluación de impacto del programa, los resultados preliminares de la evaluación mostraron avances en educación, salud y en consumo de alimentos y útiles escolares. Continúa siendo fundamental que los sectores de salud y educación fortalezcan la calidad de los servicios prestados, para que el mayor uso de los servicios se traduzca en mejoras en salud y educación y en mayor acumulación de capital humano. Los resultados obtenidos de esta evaluación arrojaron los siguientes resultados:

Pobreza: La tasa de pobreza se redujo en tres puntos porcentuales (p.p.) y el consumo per cápita aumentó en alrededor de diez por ciento, mientras que la brecha de pobreza se redujo en 2,6 p.p., y la brecha de pobreza extrema en tres p.p. Los datos oficiales más recientes (mayo 2013) mostraron una reducción en la pobreza y desigualdad: La pobreza bajó de $66,5 \%$ a $64,5 \%$ entre 2012 y 2013 ; para esos mismos años la pobreza extrema bajó de $46,0 \%$ a 42,6\% y el índice de Gini ${ }^{8}$ pasó de 0,57 a 0,54 respectivamente.

Educación: Hubo un aumento en la asistencia en el segundo y tercer ciclo de la educación básica, con impactos de 6,2 y 4,3 p.p. respectivamente. Los impactos en educación son menores cuando

\footnotetext{
8 Es una medida de la desigualdad ideada por el estadístico italiano Corrado Gini, cuyo índice se ubica entre 0 y 1 , en donde 0 se

corresponde con la perfecta igualdad (todos tienen los mismos ingresos) y donde el valor 1 se corresponde con la perfecta desigualdad (una persona tiene todos los ingresos y los demás ninguno).
}

hay más de un menor en edad escolar: en estos casos con que un menor asistiera a la escuela la familia recibía el bono completo.

Salud y nutrición: Sus efectos fueron limitados: Para niños menores de tres años aumentó en 2,6 el porcentaje de niños cuya última visita al centro de salud fue para control. Los limitados impactos en salud pueden explicarse, en parte, a que las corresponsabilidades en salud sólo aplicaban para los hogares sin niños en edad escolar. En salud materna: Hubo impactos entre las mujeres que viven en hogares en que no había niños en edad escolar (hay un efecto positivo de 12 p.p. en inmunización contra tétanos y de 22 p.p. en controles postparto) 1

Auditoría social: Se reconoció la importancia del PTMC, tanto por el apoyo que representa para el consumo de las familias, como por su efecto en motivar el uso de los servicios de salud y educación. La auditoría recomendó: i.- Mejorar la eficiencia del proceso de pagos, ii.- Crear instancias de atención al público para resolver quejas y denuncias, iii.- Promover la transparencia en las certificaciones, iv.Acercar los servicios de salud, y v.- Focalizar en la pobreza extrema rural. Dentro de las recomendaciones que se hicieran post evaluación se han identificado una agenda de ajustes a fin de mejorar la eficiencia y efectividad del programa: i.- Mejoras en la focalización del programa, ii.- Asegurar la continua verificación de corresponsabilidades y frecuencia de pago iii.- Ajuste en la estructura de corresponsabilidades, iv.- promoción de la adopción de medios bancarios y electrónicos para hacer efectivo el pago, v.- consolidación de la estrategia de género y diversidad, vi.creación de un único sistema de indicadores del programa. Vii.- homologación de los procesos de auditoria.

\section{Caracterización del Programa de Transferencia Monetaria: Bono 10 mil"}

A partir de la operacionalización de los datos obtenidos por medio de las Encuestas de Revista Economía y Administración (E\&A) / VOL. 8117 
Hogares de Propósitos Múltiples del 20122014, años en los cuales se agregó información del Bono $10 \mathrm{mil}$, fue posible su análisis dentro del contexto del impacto que este ha tenido en la población hondureña. Hay que rescatar que la focalización del programa Bono $10 \mathrm{mil}$ ha ido mejorando desde su etapa de implementación hasta la fecha, lo que implica haber hecho esfuerzos para llevar dicho bono a la población más necesitada. Otro dato importante a considerar es que en relación a la cobertura que ha tenido el Programa Bono $10 \mathrm{mil}$ en el 2012 llegó a 1,482.985 habitantes hondureños, en el 2013 a $1,764.791$ y para el 2014 alcanzó a 1,734.176 hondureños, lo que incremento en esos 3 años 251,191 hondureños, ampliando su cobertura a nivel nacional.

\subsubsection{Bono 10 mil urbano-rural}

La cobertura que el Programa Bono 10 mil ha tenido en las diferentes regiones del país, muestra que ha tenido una participación tanto en zonas urbanas como rurales, lo que implica que su impacto ha sido a nivel nacional, pero si se ve los datos desagregados, se observa la tabla No. 3 de las áreas urbanas en la que aumentó el bono en esos 3 años fue el Distrito Central con un aumento porcentual de 5.5\%, le sigue San Pedro Sula más moderadamente. Precisamente las ciudades más importantes y desarrolladas de Honduras, que convierten el corredor central de desarrollo del país es una zona cubierta por el Programa Bono 10 mil y no otras ciudades que son más pequeñas, pero con menos desarrollo.

Tabla No. 3: Hogares que reciben el bono 10 mil a nivel de dominio, 2012-2014

\begin{tabular}{|l|r|r|r|r|r|r|r|r|r|}
\hline \multirow{2}{*}{ Dominio } & \multicolumn{2}{|c|}{2012} & \multicolumn{3}{c|}{2013} & \multicolumn{3}{c|}{2014} \\
\cline { 2 - 11 } & \multicolumn{1}{|c|}{$\mathrm{Si}$} & \multicolumn{1}{c|}{ No } & \multicolumn{1}{c|}{ Total } & \multicolumn{1}{c|}{$\mathrm{Si}$} & \multicolumn{1}{c|}{ No } & Total & \multicolumn{1}{c|}{$\mathrm{Si}$} & \multicolumn{1}{c|}{ No } & Total \\
\hline Distrito Central & 7,7 & 13,5 & 12,4 & 10 & 13,5 & 12,9 & 12,8 & 14,2 & 13,9 \\
San Pedro Sula & 6,5 & 7,9 & 7,7 & 7,5 & 8 & 7,9 & 7 & 8,5 & 8,2 \\
Resto urbano & 2,4 & 30,9 & 25,8 & 2,8 & 34,9 & 25,9 & 13,7 & 35,6 & 31 \\
Rural & 83,4 & 47,7 & 54,1 & 79,7 & 43,6 & 53,3 & 66,5 & 41,7 & 46,9 \\
\hline Total & $\mathbf{1 0 0}$ & $\mathbf{1 0 0}$ & $\mathbf{1 0 0}$ & $\mathbf{1 0 0}$ & $\mathbf{1 0 0}$ & $\mathbf{1 0 0}$ & $\mathbf{1 0 0}$ & $\mathbf{1 0 0}$ & $\mathbf{1 0 0}$ \\
\hline
\end{tabular}

Fuente: Encuesta de Hogares de Propósitos Múltiples 2012-2014, INE.

La presencia del bono en zonas rurales, que son más postergadas que las ciudades han tenido prioridad, sin embargo, en el período su cobertura pasó de un $83.4 \%$ a un $66,5 \%$, lo que implica que el bono ha ido destinándose para otras zonas no tan rurales. En este sentido, haciendo un balance del impacto del bono, se puede observar que se le dio prioridad a las zonas rurales en un inicio, pero que lo han ido re direccionándolo a otras zonas del país más urbanas.

\section{Bono 10 mil a nivel departamental}

La presencia del bono a nivel departamental está enfocada en Francisco Morazán, Lempira y Cortés en un primer plano con valores superan el $13.1 \%$,
11.4 y $10.6 \%$ respectivamente, coincidente con la tendencia de la presencia del bono en el corredor central de desarrollo del país, y Lempira por ser un departamento tradicionalmente pobre se ha beneficiado del bono. En un segundo plano de importancia, Olancho con $8.9 \%$ y Choluteca con 8.5 han sido el segundo grupo de departamentos favorecidos con el bono. (Ver tabla No 4).

Sin embargo, departamentos que se han conocido por ser altamente pobres y con grandes carencias y vulnerabilidad social y económica no ha sido prioritarios para el bono, estos departamentos son: Colón, Ocotepeque y La Paz.

El único departamento que al inicio del programa 
tuvo poca presencia fue El Paraíso, pero al 2014 mejoró su participación pasando de un $2.6 \%$ en el 2012 a un 7\%. Y los departamentos que no fueron considerados al inicio del programa fueron Islas de la Bahía y Gracias a Dios.

Por otro lado, los criterios de elegibilidad de las poblaciones objetivos del programa dependen de muchos factores contingenciales, factores sociales, económicos, políticos, culturales, pero sobre todo de los mecanismos institucionales y sectoriales que acompañan al programa, lo que implica que la focalización no siempre es sobre las características ideales que se requieren, influyen factores inexorablemente exógenos.

Tabla No 4: Personas que recibieron el bono 10 mil a nivel departamental, 2012-2014 (en \%)

\begin{tabular}{|c|c|c|c|c|c|c|c|c|c|}
\hline \multirow{2}{*}{ Departamento } & \multicolumn{2}{|c|}{2012} & \multirow[b]{2}{*}{ Total } & \multicolumn{3}{|c|}{2013} & \multicolumn{3}{|c|}{2014} \\
\hline & $\mathbf{S i}$ & No & & $\mathbf{S i}$ & No & Total & $\mathbf{S i}$ & No & Total \\
\hline Atlantida & 4,3 & 5,5 & 5,3 & 2,9 & 4,8 & 4,4 & 5,7 & 6,2 & 6,1 \\
\hline Colon & 1,9 & 4,5 & 4,1 & 2,4 & 4,4 & 4 & 1,8 & 4,4 & 4 \\
\hline Comayagua & 5,2 & 5,9 & 5,7 & 4,8 & 5,4 & 5,3 & 3,7 & 3,9 & 3,8 \\
\hline Copan & 5,2 & 3,9 & 4,2 & 5,1 & 4,3 & 4,5 & 3,1 & 4,3 & 4,1 \\
\hline Cortes & 10,6 & 20,4 & 18,6 & 11,1 & 21 & 18,9 & 11,8 & 19,8 & 18,6 \\
\hline Choluteca & 8,5 & 5,2 & 5,7 & 6,7 & 5,3 & 5,6 & 9,6 & 7,6 & 7,9 \\
\hline E1 Paraiso & 2,6 & 5,6 & 5,1 & 6 & 5,1 & 5,2 & 7 & 4,7 & 5,1 \\
\hline Francisco M & 13,1 & 19,7 & 18,5 & 15,9 & 20,3 & 19,4 & 10,7 & 11,6 & 11,5 \\
\hline Gracias a Di & & & & & & & 0,9 & 0,1 & 0,2 \\
\hline Intibuca & 5,8 & 2,4 & 3 & 5 & 2,1 & 2,7 & 3,7 & 2,5 & 2,6 \\
\hline Islas de la B & & & & & & & 0 & 0,6 & 0,5 \\
\hline La Paz & 3,8 & 2,3 & 2,6 & 3,9 & 2 & 2,4 & 1,3 & 1,4 & 1,4 \\
\hline Lempira & 11,4 & 2,4 & 4 & 8,9 & 2,2 & 3,6 & 3,6 & 2,7 & 3,3 \\
\hline Ocotepeque & 2,7 & 1,5 & 1,7 & 2,2 & 1,8 & 1,9 & 0,5 & 1,8 & 1,6 \\
\hline Olancho & 8,9 & 5,9 & 6,5 & 10 & 5,8 & 6,7 & 17,4 & 8,5 & 9,8 \\
\hline Santa Bárba & 4,3 & 5,6 & 5,4 & 4,6 & 6,1 & 5,8 & 5,7 & 7,1 & 6,9 \\
\hline Valle & 4,7 & 1,9 & 2,4 & 3,3 & 2 & 2,3 & 2,5 & 2,5 & 2,5 \\
\hline Yoro & 7 & 7,3 & 7,2 & 7,4 & 7,5 & 7,5 & 7,1 & 7,7 & 7,6 \\
\hline Pase a otro & & & & & & & 1,5 & 2,6 & 2,4 \\
\hline Total & 100 & 100 & 100 & 100 & 100 & 100 & 100 & 100 & 100 \\
\hline
\end{tabular}

Fuente: Encuesta de Hogares de Propósitos Múltiples 2012-2014, INE.

\section{Bono 10 mil por condición de actividad económica}

Al observar la PEA y el comportamiento del bono, se puede observar que los ocupados recibieron el bono de manera creciente, pasaron de $47.2 \%$ a $60 \%$, una participación en el programa sumamente alta, considerando que estaban trabajando en alguna actividad económica, y los inactivos como segundo grupo, pero de manera decreciente, ya que pasaron de porcentajes de $52 \%$ a $47.4 \%$, , sin embargo, el grupo que debió haber recibido el bono de manera más consistente en el tiempo fueron los desocupados, porque obviamente se concentran la población que no está trabajando, con apenas un aumento de un $1 \%$ porcentual, lo cual es mínima su participación del bono. 
Tabla No. 5: Actividad económica del jefe de hogar, 2012-2014 (en porcentajes)

\begin{tabular}{|c|c|c|c|c|c|c|c|c|c|}
\hline \multirow{2}{*}{ Condición de Actividad } & \multicolumn{2}{|c|}{2012} & \multirow[b]{2}{*}{ Total } & \multicolumn{3}{|c|}{2013} & \multicolumn{3}{|c|}{2014} \\
\hline & Si & No & & Si & No & Total & Si & No & Total \\
\hline Ocupados & 47,2 & 49,3 & 48,9 & 48,2 & 52,4 & 51,6 & 50 & 53 & 53,1 \\
\hline Desocupac & 0,9 & 2 & 1,8 & 1,5 & 2,2 & 2,2 & 2 & 3,2 & 3 \\
\hline Inactivos & 51,9 & 48,7 & 49,2 & 50,3 & 45,4 & 46,3 & 47,4 & 43,1 & 43,9 \\
\hline Total & 100 & 100 & 100 & 100 & 100 & 100 & 100 & 100 & 100 \\
\hline
\end{tabular}

El grupo de los desocupados representan los desempleados, y los más desfavorecidos, sin embargo, son los que no fueron ubicados dentro del bono como beneficiarios principales. Situación preocupante, porque deja ver que el bono no está llegando a los más necesitados y con mayores carencias sociales. (ver tabla No.5)

\section{Bono 10 mil según nivel educativo del jefe de hogar}

El nivel educativo del jefe hogar de aquellos hogares que recibieron el bono es importante considerarlo en este análisis, el nivel que este alcanza en el sistema educativo formal impacta a dos niveles: i.- el tipo de trabajo que tiene y ii.- el ingreso que percibe por ese trabajo, lo que aseguraría la sobrevivencia de su núcleo familia - sin dejar de lado que otros miembros de la familia pueden contribuir a los ingresos familiares-.

En la tabla No. 5 muestra que los dos niveles educativos del jefe de hogar que reciben mayoritariamente el bono es el nivel básico que mantuvo su porcentaje de participación en alrededor del $57 \%$, otro grupo importante que recibe bono en segundo son aquellos jefes de hogar que no tienen ningún nivel de instrucción que pasaron de $33 \%$ a $27.7 \%$ mostrando una tendencia decreciente.

Otro grupo de jefes de hogar que reciben el bono tienen educación del Ciclo Común y Diversificado que pasaron de un porcentaje de participación de $4.7 \%$ y $4.4 \%$ en el 2012 respectivamente a $6.2 \%$ y $7 \%$ para el 2014. (ver tabla No. 6)

Tabla No. 6: Bono 10 mil por nivel educativo del jefe de hogar, 2012-2014 (en porcentajes)

\begin{tabular}{|l|r|r|r|r|r|r|r|r|r|}
\hline & \multicolumn{2}{|c|}{$\mathbf{2 0 1 2}$} & \multicolumn{3}{|c|}{$\mathbf{2 0 1 3}$} & \multicolumn{3}{|c|}{$\mathbf{2 0 1 4}$} \\
\cline { 2 - 10 } & \multicolumn{1}{|c|}{ Si } & \multicolumn{1}{|c|}{ No } & Total & \multicolumn{1}{c|}{ Si } & \multicolumn{1}{c|}{ No } & Total & \multicolumn{1}{c|}{ Si } & \multicolumn{1}{c|}{ No } & Total \\
\hline Ninguno & 33 & 26,8 & 27,8 & 30,1 & 25,5 & 26,4 & 27,7 & 24,1 & 24,8 \\
\hline Alfabetización & 0,1 & 0 & 0,1 & 0,4 & 0,2 & 0,3 & 0,4 & 0,2 & 0,3 \\
\hline Pre-básica & 0,1 & 0,1 & 0,1 & 0,2 & 0,1 & 0,1 & 0,1 & 0,2 & 0,2 \\
\hline Básica & 57,5 & 46,5 & 48,3 & 58 & 45,4 & 47,8 & 57,6 & 44,4 & 46,8 \\
\hline Ciclo común & 4,7 & 8 & 7,5 & 5,1 & 8 & 7,4 & 6,2 & 8,6 & 8,2 \\
\hline Diversificado & 4,4 & 13,9 & 12,4 & 5,6 & 15,3 & 13,4 & 7 & 15,9 & 14,3 \\
\hline Técnico superior & 0 & 0,1 & 0,1 & 0 & 0,1 & 0,1 & 0,1 & 0,1 & 0,1 \\
\hline Superior no universitaria & 0 & 0 & 0 & 0 & 0 & 0 & 0 & 0,1 & 0,1 \\
\hline Superior Universitaria & 0,2 & 4,1 & 3,5 & 0,3 & 4,9 & 4 & 0,8 & 6 & 5 \\
\hline Post-grado & 0 & 0,2 & 0,2 & 0 & 0,2 & 0,2 & 0 & 0,2 & 0,2 \\
\hline No sabe/no responde & 0 & 0,2 & 0,2 & 0,2 & 0,3 & 0,3 & 0,2 & 0,2 & 0 \\
\hline Total & 100 & 100 & 100 & 100 & 100 & 100 & 100 & 100 & 100 \\
\hline
\end{tabular}

Fuente: Encuesta de Hogares de Propósitos Múltiples 2012-2014, INE. 
Dentro del Programa del Bono 10 mil hay dos segmentos de jefes de hogar, i, Jefes sin ninguna instrucción y que van más acorde con los requerimientos de participación del bono y ii.Jefes de hogar con niveles de educación básica y media, lo que implica una polarización de los grupos prioritarios del programa.

\section{Bono 10 mil según clasificación de pobreza}

Los niveles de pobreza de aquellos hogares que participan del programa bono 10 mil se manejan bajo el criterio del acceso a la canasta básica de alimentos y canasta básica se acuerdo con la Encuesta de Hogares de Propósitos Múltiples, lo que deja ver que efectivamente el bono 10 mil esta llegado a los de extrema pobreza o sea aquellos hogares que no pueden comprar ni la canasta básica de alimentos, ni la canasta básica, lo que viene a beneficiar la situación de ingresos a los hogares de esta condición con porcentajes de participación en el programa que va de $68.5 \%$ en el 2012 a $62.2 \%$ en el 2014, de forma decreciente pero manteniendo su priorización dentro del programa. (Ver tabla No. 7).

Tabla No. 7: Hogares que recibieron el Bono 10 mil según clasificación de la pobreza

\begin{tabular}{|c|c|c|c|c|c|c|c|c|c|}
\hline Clasificación de & 2012 & & & 2013 & & & 2014 & & \\
\hline la pobreza & $\mathbf{S i}$ & No & Total & Si & No & Total & $\mathbf{S i}$ & No & Tota \\
\hline Extrema & 68,5 & 42,3 & 46 & 66,5 & 38 & 42,6 & 62,2 & 35,3 & 39,7 \\
\hline Relativa & 15,9 & 21,3 & 20,5 & 19,2 & 22,5 & 21,9 & 19,9 & 23,7 & 23,1 \\
\hline No pobres & 15,6 & 36,4 & 33,5 & 14,3 & 39,5 & 35,5 & 17,9 & 41 & 37,2 \\
\hline Total & 100 & 100 & 100 & 100 & 100 & 100 & 100 & 100 & 100 \\
\hline
\end{tabular}

Sin embargo, también los hogares con una pobreza relativa, o sea aquellos hogares que solo pueden comprar la canasta básica de alimentos, incrementó levemente su participación del bono, paso de $15.0 \%$ a $19.9 \%$. Asimismo, los no pobres también pasaron de $15.6 \%$ a $17.9 \%$, implica en términos generales fueron considerados no solo los más pobres y vulnerables, sino también aquellos hogares con algunas mejores condiciones de vida, pero que eran elegibles para participar en el programa.

\section{Bono 10 mil por ingreso mensual del hogar}

El ingreso mensual fueron otras variables para que la participación de los hogares en el Bono 10 mil, se ve claramente que los hogares más favorecidos con el bono fueron aquellos hogares que tienes ingresos menores del salario mínimo, menos de L.
7,500.00 sin embargo, se dio un decrecimiento en este grupo de hogares, pasó de 5.9\%. en, el 2012 a $65 \%$ en el 2014. Aquellos hogares que tenían ingresos mensuales entre L. 7,500.00 a 12,500.00 y que recibían el bono pasaron de $17.8 \%$ a $18.2 \%$ en el período, una leve mejoría en la participación del bono. (Ver tabla No. 8

Generalmente los hogares con ingresos menores de L. 12,500.00 reciben el bono $10 \mathrm{mil}$, lo que implica que el programa está llegando aquellos hogares con menor capacidad de ingresos y por consiguiente mayores necesidades existenciales y básicas. Cabe destacar que en los hogares con ingresos menores de L7,500.00 y hasta L.12,500.00 son lo que tienen concentrados el beneficio del bono superando el $83.6 \%$, una alta concentración de los beneficios del programa. 
Tabla No. 8: Ingreso mensual de los hogares que recibieron el bono 10 mil, 2012-2014, (en porcentajes)

\begin{tabular}{|l|r|r|r|r|r|r|r|r|r|}
\hline \multirow{2}{*}{ Ingreso Mensual } & \multicolumn{2}{|c|}{$\mathbf{2 0 1 2}$} & \multicolumn{3}{|c|}{$\mathbf{2 0 1 3}$} & \multicolumn{3}{|c|}{$\mathbf{2 0 1 4}$} \\
\cline { 2 - 11 } & \multicolumn{1}{|c|}{ Si } & \multicolumn{1}{|c|}{ No } & Total & \multicolumn{1}{c|}{ Si } & No & Total & \multicolumn{1}{c|}{ Si } & \multicolumn{1}{c|}{ No } & Total \\
\hline Menos de L.7,500.00 & 75,9 & 59,4 & 61,8 & 72,1 & 54,7 & 57,5 & 65,4 & 50,9 & 53,3 \\
\hline De L. 7,501 - 12,500 & 14,8 & 17,8 & 17,4 & 18,3 & 20,1 & 19,8 & 18,2 & 20,8 & 20,4 \\
\hline De L. 12,501 - 17,500 & 4,4 & 8,7 & 8,1 & 5,2 & 9,9 & 9,2 & 9 & 11,4 & 10,9 \\
\hline De L. 17,501 - 22,500 & 2,5 & 5,1 & 4,8 & 1,9 & 5,2 & 4,7 & 4,1 & 6 & 5,7 \\
\hline De L. 22,501 - 32,500 & 1,6 & 4,3 & 3,9 & 1,8 & 2,5 & 4,8 & 2,3 & 5,5 & 4,9 \\
\hline De L. 32,501 - 42,500 & 0,3 & 2 & 2,1 & 0,5 & 2,1 & 1,9 & 0,7 & 2,4 & 2,1 \\
\hline Mas de L. 42,501 & 0,5 & 2,7 & 1,9 & 0,3 & 0,2 & 2,1 & 0,3 & 3 & 2,7 \\
\hline Total & & & & & & & & & 100 \\
\hline
\end{tabular}

Fuente: Encuesta de Hogares de Propósitos Múltiples 2012-2014, INE.

Generalmente los hogares con ingresos menores de L. 12,500.00 reciben el bono $10 \mathrm{mil}$, lo que implica que el programa está llegando aquellos hogares con menor capacidad de ingresos y por consiguiente mayores necesidades existenciales y básicas

\section{Bono 10 mil según sexo del jefe de hogar}

Las jefaturas de hogar de hombres que han sido beneficiados por el bono 10 mil están concentradas en las áreas rurales pero de manera decreciente, ya que han pasado de $58 \%$ a $51.4 \%$ del 2012 al 2014 , implica un dominio de hombres jefes como proveedores principales de sus hogares. (ver tabla No. 9)

Sin embargo, en zonas urbanas el predominio de jefaturas de hogar la tiene la mujer con porcentajes que superar el $30 \%$ en el período, tanto en el Distrito Central con valores arriba del $15 \%$ y como el resto urbano. Que las mujeres estén migrando más a las ciudades y en condiciones de desventajas y con grandes limitaciones económicas y sociales.

Tabla No. 9: Bono 10 mil por sexo del jefe de hogar, según dominio de residencia, 2012-2014 (en porcentajes)

\begin{tabular}{|l|r|r|r|r|r|r|r|r|r|}
\hline \multirow{2}{*}{ Dominio } & \multicolumn{2}{|c|}{$\mathbf{2 0 1 2}$} & \multicolumn{3}{c|}{$\mathbf{2 0 1 3}$} & \multicolumn{3}{c|}{$\mathbf{2 0 1 4}$} \\
\cline { 2 - 11 } & \multicolumn{1}{|c|}{ H } & M & Total & \multicolumn{1}{c|}{ H } & M & Total & H & M & \multicolumn{1}{c|}{ Total } \\
\hline Distrito Central & 10,9 & 16,2 & 12,4 & 11 & 17,1 & 12,9 & 12,1 & 17,6 & 13,9 \\
\hline San Pedro Sula & 7,5 & 8 & 7,7 & 7,7 & 8,5 & 7,9 & 7,7 & 9 & 8,2 \\
Resto urbano & 23,6 & 31,2 & 25,8 & 23,9 & 59,4 & 25,9 & 28,8 & 35,7 & 31 \\
Rural & 58 & 44,6 & 54,1 & 57,4 & 44,1 & 53,3 & 51,4 & 37,7 & 46,9 \\
\hline Total & $\mathbf{1 0 0}$ & $\mathbf{1 0 0}$ & $\mathbf{1 0 0}$ & $\mathbf{1 0 0}$ & $\mathbf{1 2 9 , 1}$ & $\mathbf{1 0 0}$ & $\mathbf{1 0 0}$ & $\mathbf{1 0 0}$ & $\mathbf{1 0 0}$ \\
\hline
\end{tabular}

Fuente: Encuesta de Hogares de Propósitos Múltiples 2012-2014, INE. 


\section{Bono 10 mil por quintil del hogar}

Analizando el ingreso por medio de quintiles, implica que los hogares beneficiarios del bono están concentrados en los tres primeros quintiles aquellos quintiles con menores ingresos, sin embargo, el quintil 1 y 2 concentran más del $60 \%$ de hogares que reciben el bono. (ver tabla No. 10).

Tabla No 10: Bono 10 mil por quintil de ingresos de los hogares, 2012-2014 (en porcentajes)

\begin{tabular}{|l|r|r|r|r|r|r|r|r|r|}
\hline Quintil & \multicolumn{2}{|c|}{$\mathbf{2 0 1 2}$} & \multicolumn{3}{c|}{$\mathbf{2 0 1 3}$} & \multicolumn{3}{c|}{$\mathbf{2 0 1 4}$} \\
\cline { 2 - 11 } & \multicolumn{1}{|c|}{ Si } & \multicolumn{1}{c|}{ No } & \multicolumn{1}{l|}{ Total } & \multicolumn{1}{c|}{ Si } & \multicolumn{1}{c|}{ No } & \multicolumn{1}{c|}{ Total } & \multicolumn{1}{c|}{ Si } & \multicolumn{1}{c|}{ No } & \multicolumn{1}{c|}{ Total } \\
\hline Quintil 1 & 34,4 & 17,5 & 19,9 & 38 & 16,5 & 19,9 & 37,3 & 19 & 22,8 \\
Quintil 2 & 29,5 & 18,3 & 19,9 & 27,5 & 18,4 & 19,9 & 28,9 & 20,1 & 21,9 \\
Quintil 3 & 20,3 & 19,8 & 19,9 & 20,8 & 19,7 & 19,9 & 17,4 & 21,1 & 20,3 \\
Quintil 4 & 11,1 & 21,4 & 19,9 & 10,3 & 21,7 & 19,9 & 13 & 20,3 & 18,9 \\
Quintil 5 & 4,7 & 22,4 & 19,9 & 3,3 & 23 & 19,9 & 3,3 & 18,9 & 15,6 \\
No declaran & 0 & 0,6 & 0,5 & 0 & 0,7 & 0,5 & 0,1 & 0,6 & 0,5 \\
& & & & & & & & & \\
\hline Total & 100 & 100 & 100 & 99,9 & 100 & 100 & 100 & 100 & 100 \\
\hline
\end{tabular}

Fuente: Encuesta de Hogares de Propósitos Múltiples 2012-2014, INE.

El programa bono 10 mil está llegando a los segmentos de población con menores ingresos, lo que implica que se cumplen los principios de elegibilidad de hogares pobres y con menos recursos económicos participando del bono 10 mil.

\section{Vinculaciones de población y las transferencias monetarias condicionadas}

\section{Bono 10 Mil por grupos de edad}

Se observa los grupos de edad de la población que participa o recibe el bono $10 \mathrm{mil}$, se observa claramente en la tabla No. 11 que el grupo de 1559 años es el grupo que se beneficia más del bono, superando este beneficio más del $60 \%$, seguido del grupo de 0-14 años de edad, que tienen una participación del bono superando el $40 \%$.

Implica la participación e jefes de hogar y sus hijos, se benefician como núcleo familiar de del programa, ya que una de las condicionalidades para participar es que en los hogares tengan hijos estudiando en la escuela, lo cual beneficia no solo la asistencia de los niños a las escuelas, sino de las visitas de control a los centros de salud de sus comunidades, logrando doble beneficios los niños y sus madres.

Sin embargo, la población mayor de 60 años y más es el grupo que menos beneficios tiene del bono, prácticamente es nula su participación, lo deja fuera como grupo etario.

Tabla No. 11: Bono 10 mil por grupos de edad, 2012-2014

(en porcentajes)

\begin{tabular}{|l|r|r|r|r|r|r|r|r|r|}
\hline \multirow{2}{*}{ Grupos de edad } & \multicolumn{2}{|c|}{$\mathbf{2 0 1 2}$} & \multicolumn{3}{|c|}{$\mathbf{2 0 1 3}$} & \multicolumn{3}{|c|}{$\mathbf{2 0 1 4}$} \\
\cline { 2 - 11 } & \multicolumn{1}{|c|}{ Si } & \multicolumn{1}{c|}{ No } & Total & \multicolumn{1}{c|}{ Si } & \multicolumn{1}{c|}{ No } & \multicolumn{1}{c|}{ Total } & \multicolumn{1}{c|}{ Si } & \multicolumn{1}{c|}{ No } & \multicolumn{1}{c|}{ Total } \\
\hline 0-14 años & 44,9 & 30,7 & 33,2 & 45,4 & 30,8 & 33,8 & 44,4 & 30,1 & 33,1 \\
15-59 años & 50,4 & 58,8 & 57,3 & 50 & 58,4 & 56,7 & 51,6 & 60,1 & 58,3 \\
\hline 60 y mas años & 4,7 & 10,5 & 9,5 & 4,6 & 10,8 & 9,5 & 4 & 9,8 & 8,6 \\
& & & & & & & & & \\
\hline Total & 100 & 100 & 100 & 100 & 100 & 100 & 100 & 100 & 100 \\
\hline
\end{tabular}

Fuente: Encuesta de Hogares de Propósitos Múltiples 2012-2014, INE. 


\section{Síntesis}

El programa Bono 10 mil en su conjunto está orientado a fortalecer y apoyar aquellos hogares que tienen mayores carencias y desventajas sociales y económicas, en su trayectoria ha logrado elevar los indicadores, producto de la intervención y del bono que mejora la capacidad adquisitiva de los hogares, sin embargo, ha habido inconsistencias, y la focalización de los hogares ha ido perfeccionándose $\mathrm{y}$ tratando de alcanzar aquellas zonas y hogares que realmente necesitan del bono.

No todos los criterios seleccionados para beneficiar dichas familias han sido las más justas y equitativas, pero los esfuerzos se han dado y muchas situaciones han mejorado, aún queda mucho que aprender y mejorar, sobre todo porque mientras el bono sea dado a las familias beneficiarias de este sus condiciones mejoraran.

Sin embargo, si los esfuerzos logrados por el Programa Bono $10 \mathrm{mil}$, no va acompañado de otros cambios sustantivos y radicales en la política social del país, es difícil que los cambios favorables que se han dado sean consistes en el tiempo y permanezca como logros sociales, desde el Estado hay que implementar políticas pública más inclusivas y favorables para los desfavorables del país, queda todo un camino por recorrer y mucho que aprender aún más. 


\section{REFERENCIAS}

Castro, M. (2007) El Monitoreo y la Evaluación de la Gestión Pública Honduras, Documentos Estudios de Casos del CLAD, No. 3, Centro Latinoamericano de Administración para el Desarrollo (CLAD), Banco Mundial, Washington.

Fonseca, A., (2006) Los sistemas de protección social en América Latina: Un análisis de las transferencias monetarias condicionadas. Seminario de transferencias condicionadas y seguridad alimentaria: puertas de salida a la pobreza extrema a través del combate al hambre. Santiago.

Instituto Nacional de Estadística (2012) Encuesta Permanente de Hogares de Propósitos Múltiples, Tegucigalpa.

Instituto Nacional de Estadística (2013) Encuesta Permanente de Hogares de Propósitos Múltiples, Tegucigalpa.

Instituto Nacional de Estadística (2014) Encuesta Permanente de Hogares de Propósitos Múltiples, Tegucigalpa.
Martínez, R. \& Collinao, M. P. (2010). Gasto social: Modelo de medición y análisis.

Paes-Sousa, R., Regalia F., \& Stampini, M. (2013). Marco condiciones para el éxito de la puesta en práctica de programas de transferencias monetarias condicionadas: lecciones de América Latina y el Caribe para Asia: Resumen de políticas del BID \# IDB-PB-192. División de Protección Social y Salud, BID.

Serrano, C. (2005). Claves de la política social para la pobreza. Manuscrito no publicado.

Tokman, V. (2003), "Hacia una visión integrada para enfrentar la inestabilidad y el riesgo", en Revista de la CEPAL, $\mathrm{N}^{\circ}$ 81, Santiago, diciembre. $\quad$ http://repositorio.cepal.org/ bitstream/handle/11362/5108/1/S34173 es.pdf

Tokman, V. (2006) "Inserción laboral, mercados de trabajo y protección social", Santiago, CEPAL (Serie Políticas Sociales). 


\section{NOTAS}




\title{
CONTEXTO INDUSTRIAL EN COMPETITIVIDAD: ESTRATEGIA DE MANUFACTURA Y TECNOLOGÍA EN PRODUCCIÓN
}

\author{
Cesar H. Ortega Jimenez \\ Universidad Nacional Autónoma de Honduras, IIES, Tegucigalpa, Honduras \\ Facultad de Ciencias Económicas, Administrativas y Contables (FCEAC) \\ Instituto de Investigaciones Económicas y Sociales (IIES-UNAH) \\ Ciudad universitaria, edificio C2, primer piso, Tel/fax: + (504) 2216-6100 Ext. 100894 \\ ORCID: 0000-0003-1911-9725 \\ cortega@unah.edu.hn \\ Iván A. Arana \\ Instituto Tecnológico y de Estudios Superiores de Monterrey, México D.F., México \\ iarana@itesm.mx \\ (1) Rafaela Alfalla \\ Universidad de Sevilla, Sevilla, España \\ alfalla@us.es \\ José Luis Pérez-Díez \\ Universidad de Sevilla, Sevilla, España \\ jlperezd@us.es
}

DOI http://dx.doi.org/10.5377/eya.v8i2.5618

\section{RESUMEN}

Las fábricas tienen que implementar programas de operaciones, con el fin de no sólo mejorar la competitividad, sino también de mantener una mejora continua que les permita enfrentar los desafíos por los cambios constantes del contexto industrial. Entre dichos programas, la gestión de tecnología (GT) y la estrategia de manufactura (EM) podrían ser un factor de importancia para mejorar la competitividad. El propósito de este estudio es verificar empíricamente la relación entre ambos programas en las industrias de maquinaria y electrónica, tomando en cuenta algunas variables contextuales. Para evaluar las hipótesis establecidas, usamos la base de datos del proyecto internacional de Manufactura de Alto Rendimiento (por sus siglas en inglés, HPM) y análisis de regresión jerárquico. Los hallazgos muestran que para ambos sectores la incorporación de GT mejoró la competitividad. Sin embargo, solo una de las variables contextuales, capacidad productiva de la fábrica, tiene una relación consistente con la competitividad en el sector de electrónica.

Palabras clave: Estrategia de manufactura, Gestión de Tecnología, Competitividad 


\title{
INDUSTRIAL CONTEXT IN COMPETITIVENESS: MANUFACTURING AND PRODUCTION TECHNOLOGY STRATEGY
}

\author{
Cesar H. Ortega Jimenez \\ Universidad Nacional Autónoma de Honduras, IIES, Tegucigalpa, Honduras \\ Facultad de Ciencias Económicas, Administrativas y Contables (FCEAC) \\ Instituto de Investigaciones Económicas y Sociales (IIES-UNAH) \\ Ciudad universitaria, edificio C2, primer piso, Tel/fax: + (504) 2216-6100 Ext. 100894 \\ ORCID: 0000-0003-1911-9725 \\ cortega@unah.edu.hn \\ Iván A. Arana \\ Instituto Tecnológico y de Estudios Superiores de Monterrey, México D.F., México \\ iarana@itesm.mx \\ (1) Rafaela Alfalla \\ Universidad de Sevilla, Sevilla, España \\ alfalla@us.es \\ José Luis Pérez-Díez \\ Universidad de Sevilla, Sevilla, España \\ jlperezd@us.es
}

DOI http://dx.doi.org/10.5377/eya.v8i2.5618

\begin{abstract}
The factories must implement operations programs, in order not only to improve competitiveness, but also to maintain a continuous improvement that allows them to face the challenges due to the constant changes in the industrial context. Among such programs, technology management (GT) and manufacturing strategy (EM) could be an important factor in improving competitiveness. The purpose of this study is to verify empirically the relationship between both programs in the machinery and electronics industries, considering some contextual variables. To evaluate the established hypotheses, we use the database of the international project of High Performance Manufacturing (HPM) and hierarchical regression analysis. The findings show that for both sectors the incorporation of GT improved competitiveness. However, only one of the contextual variables, productive capacity of the factory, has a consistent relationship with competitiveness in the electronics sector.
\end{abstract}

Keywords: Manufacturing strategy, Technology Management, Competitiveness

(1) Corresponding author

128 Revista Economía y Administración (E\&A) / VOL. 8 


\section{INTRODUCCIÓN}

Losfabricantes enfrentan presiones sin precedentes de productos extranjeros, presentación de productos nuevos por la competencia, innovación tecnológica rápida y productos de corta duración, cambios imprevistos del consumidor y avances en manufactura e información tecnológica. Para enfrentar las amenazas ambientales, las fábricas tienen que implementar programas de operaciones (PO) (i.e. conjunto de prácticas avanzadas de operaciones agrupadas para un resultado común de la gestión de una organización) para aumentar la competitividad. La gestión de tecnología (GT) es un programa importante para mejorar la competitividad (medido por el rendimiento operativo/RO). Sin embrago, de tener que implementar la GT, la falta de estrategias de manufactura (EM) que no sólo integre las áreas funcionales de la fábrica, sino que también sea consistente con la estrategia de negocios, podría conllevar a una poca competitividad en la fábrica. De hecho, varios estudios han hallado una interrelación positiva entre EM y RO en diferentes tipos de industrias y países (v.g. Morita and Flynn, 1997; Devaraj et al., 2004; Corbett, 2008; da Silveira and Sousa, 2010, Machuca et al., 2011; Lee, 2014).

Actualmente, los fabricantes están usando cada vez más las tecnologías más avanzadas. La tendencia está impulsada por el argumento que el uso de la tecnología conducirá al mejoramiento en algunas medidas del $\mathrm{RO}$, como la reducción de costos laborales o mejorar la calidad y flexibilidad (Heine et al., 2003). Sin embargo, en muchos casos, estas inversiones no han dado el resultado deseado. Esto es porque el vínculo entre GT y rendimiento está altamente influenciado por factores contingentes (Malhotra et al., 2001). De este modo, la existencia simple de tecnología no es suficiente, debe estar acompañada por variables contingentes para que sea efectiva (Heine et $a l ., 2003)$. En la literatura, se ha encontrado evidencia positiva que la GT mejora el RO (Boyer et al., 1996; Flynn and Flynn, 1999; Maier and Schroeder, 2001; Tsai, 2004; Raymond, 2005; Machuca et al., 2011). Sin embargo, algunos dios han concluido que la GT no influye en el RO (Beaumont and Schroeder, 1997; Boyer et al., 1997; Swamidass and Kotha, 1998; Cagliano and Spina, 2000; Das and Jayaram, 2003) debido a la variedad de factores contingentes. Dichas contradicciones implican la necesidad de mayor y nueva evidencia empírica en la interrelación entre GT y RO tomando en cuenta las variables contextuales.

Mientras la influencia de prácticas operacionales en el rendimiento es inherentemente interesante en el contexto de un país en vías de desarrollo, también estamos interesando en entender si esta relación depende de las variables contextuales (Heine et al., 2003). Específicamente, del mismo modo se desea investigar si las variables contextuales afectan la relación entre EM, GT y RO. Además, a pesar de la aparente relación entre EM y GT, pocos estudios han investigado como afectan actualmente la influencia de variables contextuales en RO (v.g. Machuca et al., 2011). Por lo tanto, existe una falta de investigación empírica en este tema.

El propósito principal de este estudio es explorar, usando una base empírica, si existe una relación positiva entre EM, GT y RO, aún con la inclusión de variables contextuales, así como si esta relación varía por el tipo de industria tanto de electrónica como de maquinaria. Los resultados además de proveer un mejor entendimiento de la relación de EM y GT, en dos contextos industriales, guiarán a los profesionales de la gestión empresarial a enfocarse mejor.

\section{II.- MARCO CONCEPTUAL E HIPÓTESIS}

Las organizaciones sepueden comportardiferentes, dependiendo de si están ubicadas en contextos estables o dinámicos. Tiene que implementar PO no solo considerando los aspectos del contexto, pero también la interconexión entre prácticas de manufactura para obtener una mejor posición competitiva (Schroeder and Flynn, 2001). Por ejemplo, en mercados volátiles, como el sector electrónico, una implementación de tecnología de alto nivel en productos y procesos puede ser un factor predominante para ser competitivos (Fine, 2000; Mallick and Schroeder, 2005). Sin 
embargo, en otro tipo de industrias, donde el mercado es más estable con productos de ciclos de larga duración, como el sector de maquinaria, EM tiene que relacionarse con otros PO para obtener ventajas competitivas (Schroeder and Flynn, 2001).

La existencia de una relación positiva entre EM y RO ha sido respaldada bastante por la literatura de estrategia de manufactura. Varios estudios han mostrado que EM está asociado con RO positivo y significativa (Morita and Flynn, 1997; Bates et al., 2001; Ketokivi and Schroeder, 2004; Devaraj et al., 2004; Amoako-Gyampah and Acquaah, 2008; Corbett, 2008; Rose et al., 2008; Thun, 2008; Oltra and Flor, 2010; da Silveira and Sousa, 2010; Machuca et al. 2011; Lee, 2014). La influencia positiva del mejoramiento de RO se atribuye parcialmente a la implementación más completa de una EM y de las variables contextuales (Papke-Shields et al., 2006; Schroeder et al., 2002). Aun los estudios empíricos de la relación entre EM y RO no prestaron mucha atención a las variables contextuales (excepto Machuca et al. (2011)) más evidencia es necesaria para evaluar las siguientes hipótesis:

\section{H1. La implementación de EM es asociada positivamente con $R O$ en la presencia de variables contextuales.}

El efecto de GT en RO presenta hallazgos no concluyentes en la literatura anterior. Los investigadores han encontrado efectos directos positivos y significativas de GT en RO (Boyer et al., 1996; FlynnandFlynn, 1999; Maier and Schroeder, 2001; Tsai, 2004; Raymond, 2005) o en medida de RO como productividad, calidad y flexibilidad (Gyan Baffour, 1994; Beede and Young, 1998), pero también existe evidencia empírica que no apoya la relación EM-RO (Beaumont and Schroeder, 1997; Boyer et al., 1997; Swamidass and Kotha, 1998; Cagliano and Spina, 2000; Das and Jayaram, 2003, Machuca et al., 2011). Algunos investigadores refieren este patrón de resultado inconcluso o variable a la influencia de factores contingentes, como el tamaño de la planta, tipo de proceso de manufactura, métodos de organización laboral y estrategia de competitividad (Sun and Gertsen, 1995, Swamidass and Kotha, 1998; Cagliano and
Spina, 2000). Sin embargo, aunque los efectos de EM en RO no son claros, hasta las variables contextuales están presentes. La siguiente hipótesis será evaluada:

H2. La implementación de GT es asociada positivamente con $R O$ en la presencia de variables contextuales.

De la misma manera, la presencia o ausencia de estas variables contextuales podrían afectar materialmente la influencia de EM o GT en resultados del RO (Im and Lee, 1989; Primrose, 1992; Groenevelt, 1993; Boyer et al., 1997, Cagliano and Spina, 2000; Ahmad et al., 2003. Machuca et al. 2011), examinaron las influencias de EM y GT en RO en el sector auto proveedor. Los resultados revelaron que la EM está relacionada positivamente con el RO y continúa haciéndolo cuando los factores contextuales están presentes. En el caso de GT, no existe una relación con el $\mathrm{RO}$, aun cuando se incluyeron las variables contextuales. Sin embargo, el estudio se enfocó en una industria específica, auto proveedora, abriendo estudios futuros para otras industrias. Es decir, se espera que la influencia de ambos conjuntos de prácticas en cuestión debiera permanecer significativa luego de que el control de variables haya sido incluido. Por lo tanto, evaluamos la siguiente hipótesis:

\section{H3. La implementación de EM y GT son asociadas positivamente con $R O$ en la presencia de variables contextuales.}

No obstante, pocos estudios se han enfocado en la contribución de variables contextuales a mejorar el RO. Consecuentemente, la investigación de la influencia de variables contextuales en el RO en el contexto de los sectores electrónicos y de maquinaria ha permanecido sin ser realizados. Usamos factores contextuales como ser tamaño de la planta (TP), capacidad de uso de la planta (UP) y personalización del producto (PP) siguiendo a Machuca et al. (2011). Son simplemente usados para evaluar la efectividad de las variables contextuales en el RO. Esto conlleva a la siguiente hipótesis:

H4. Las variables contextuales son asociadas positivamente con $R O$. 


\section{III.- METODOLOGÍA}

\section{III.1.- Muestreo y recolección de datos}

El análisis empírico de este trabajo está basado en el proyecto de base de datos de HPM (siglas en inglés, Manufactura de Alto Rendimiento) internacional. La técnica de investigación usada para obtener los datos fue una encuesta. La base de datos cubre industrias maquinarias y electrónica en nueve países (Austria, Canadá, Finlandia, Alemania, Italia, Japón, Corea, España, Suecia, y los Estados Unidos) de 176 plantas con más de 100 empleados, divididos en 88 del sector de maquinaria y 88 del sector de la electrónica.

En total, se usaron 12 tipos de cuestionarios, dirigidos a varios encuestados en cada planta (desde gerente de planta hasta trabajadores) y presentado a 21 encuestados. Todas las escalas y medidas de prácticas demanufacturas consideradas en el proyecto de HPM fueron incluidas en estos cuestionarios. Tres diferentes investigadores desarrollaron y examinaron los artículos para las escalas con el propósito de asegurar la validez del contenido de los cuestionarios.

Los cuestionarios fueron probados usando expertos y académicos de la industria, y los artículos estaban incluidos en al menos dos diferentes cuestionarios, con el objetivo de triangular información para mayor fiabilidad. Esto da una imagen transversal de la planta para evitar el prejuicio individual (Van Bruggen et al., 2002; Sakakibara et al., 1997) mientras se aumenta simultáneamente la validez. Los artículos y preguntas que constituye cada escala fueron mezclados en cada cuestionario para evitar posibles prejuicios en el encuestado.
Los cuestionarios fueron examinados durante la primera y segunda ronda del proyecto de HPM y fueron traducidos y luego traducidos de vuelta por diferentes individuos para verificar la precisión. Cualquier diferencia identificada durante este proceso fue resuelta antes del lanzamiento de las encuestas a países no angloparlantes. Como resultado, la consistencia interna, la validez del contenido y la validez de constructo tienen valores altos en la escala final usada (Amahad and Schroeder, 2002; Schroeder and Flynn, 2001; Flynn et al., 1995, Machuca et al., 2011).

\section{III.2.- Escalas}

Los artículos usados en la presente investigación fueron dirigidos a nivel de planta para evaluar la consistencia interna. EM y GT fueron evaluados perceptualmente, usando una escala Likert de siete puntos, que van desde 1 = muy en desacuerdo a 7 = muy de acuerdo. La fiabilidad fue medida por valores alfa de Cronbach mayores de 0.6 (Hair et al., 2011), suponiendo que fueron consistentes internamente. La Tabla 1 muestra los valores obtenidos el alfa de Cronbach (Cronbach, 1951) y su intervalo de confianza (Koning and Franses, 2003) para cada escala e industria. Por lo tanto, el constructo EM tiene un alfa de Cronbach de 0.829 y 0.779 en las áreas de maquinaria y electrónica, respectivamente. GT tiene un alfa de Cronbach de 0.651 y 0.625 en las áreas de maquinaria y electrónica, respectivamente.

RO fue medido usando la escala de Likert de cinco-puntos, que van desde $1=$ deficiente, entre lo peor de la industria a $5=$ superior. El constructo RO tiene un alfa de Cronbach de 0.656 y 0.736 en las áreas de maquinaria y electrónica, respectivamente. 
Tabla No. 1 - Fiabilidad de las Escalas

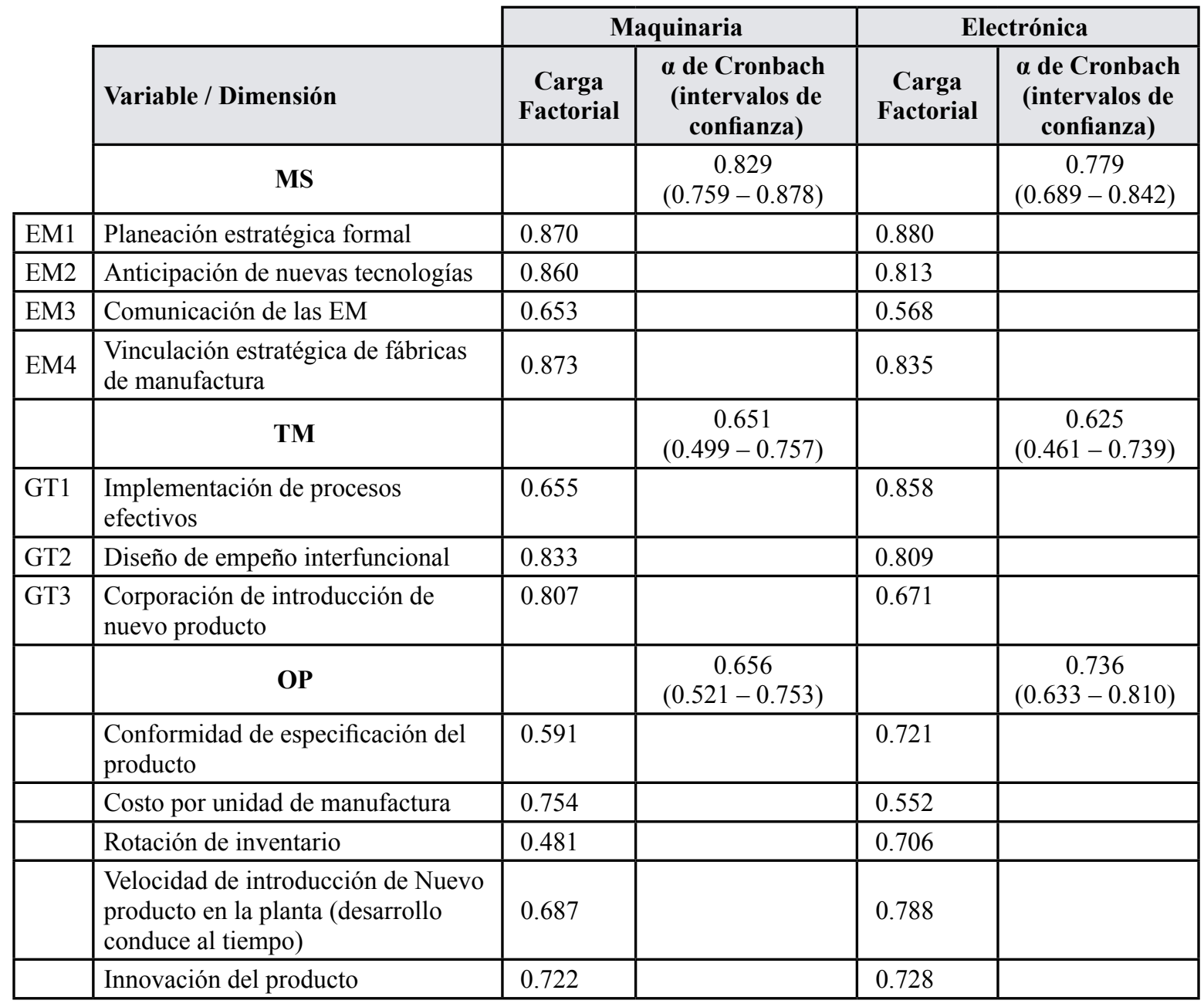

Se incluyen factores contextuales como uso de la planta (UP), tamaño de la planta (TP) y personalización del producto (PP). El uso de todos los factores contextuales afectando el rendimiento no es posible, por lo que lo limitamos a tres, siguiendo a Ahmad et al. 2003 y Machuca et al. 2011. La Tabla 2 muestra valores promedios de tres variables contextuales por área.

Tabla No. 2 - Información descriptiva de variables contextuales

\begin{tabular}{|l|c|c|}
\hline \multicolumn{1}{|c|}{ Variable } & Maquinaria & Electrónica \\
\hline TP: Tamaño de la planta (número de empleados) & 1045 & 593 \\
\hline UP: Porcentaje promedio de capacidad de uso de la planta (\%) & 86.38 & 83.21 \\
\hline PP: Grado de porcentaje de personalización del producto & 2.94 & 3.18 \\
\hline
\end{tabular}




\section{III.3.- Evaluación del modelo}

El análisis de la regresión jerárquica fue usado para evaluar la hipótesis. Este análisis permitió al investigado identificar el porcentaje de varianza explicado de cada variable independiente de manera separada (Pedhazur and Schmelkin, 1991; Cagliano, et al., 2006). De hecho, la división de varianza por medio del análisis de la regresión jerárquica es la metodología más apropiada cuando existen correlaciones entre las variables independientes. Las correlaciones entre las variables consideradas en el análisis se muestran en la Tabla 3 (plantas maquinarias) y en la Tabla 4 (plantas electrónicas). Se debe remarcar que existen correlaciones importantes entre las variables contextuales, EM, GT y RO, de este modo es considerado para análisis futuros.

Tabla No. 3 - Resultados de correlación de plantas maquinarias

\begin{tabular}{|l|c|c|c|c|c|c|c|c|}
\cline { 2 - 9 } \multicolumn{1}{c|}{} & Mean & S.D. & 1 & 2 & 3 & 4 & 5 & 6 \\
\hline $\begin{array}{l}\text { Capacidad } \\
\text { productiva de la } \\
\text { fábrica }\end{array}$ & 85.591 & 13.325 & 1 & -0.017 & 0.22 & 0.169 & 0.219 & 0.238 \\
\hline $\begin{array}{l}\text { Tamaño de la } \\
\text { Planta }\end{array}$ & 5.892 & 0.954 & & 1 & -0.059 & 0.032 & -0.13 & -0.016 \\
\hline $\begin{array}{l}\text { Personalización } \\
\text { del producto }\end{array}$ & 3.333 & 2.147 & & & 1 & $.339^{* *}$ & $.270^{*}$ & 0.179 \\
\hline EM & 5.045 & 0.623 & & & & 1 & $.669^{* *}$ & $.352^{* *}$ \\
\hline GT & 4.864 & 0.607 & & & & & 1 & $.451^{* *}$ \\
\hline RO & 3.530 & 0.547 & & & & & & 1 \\
\hline
\end{tabular}

${ }^{*} P \leq 0.1 ; * * P \leq 0.05 ; * * * P \leq 0.01$

Tabla No. 4 - Resultados de correlación de plantas electrónicas

\begin{tabular}{|l|c|c|c|c|c|c|c|c|}
\cline { 2 - 9 } \multicolumn{1}{c|}{} & Mean & S.D. & PU & PS & PC & MS & TM & OP \\
\hline UP & 83.215 & 15.891 & 1 & -0.215 & 0.199 & 0.012 & -0.108 & $.289^{* *}$ \\
\hline TP & 5.931 & 0.965 & & 1 & -0.109 & $.292^{* * *}$ & $.381^{* * *}$ & 0.212 \\
\hline PP & 3.184 & 1.798 & & & 1 & 0.071 & 0.00 & -0.088 \\
\hline EM & 5.243 & 0.571 & & & & 1 & $.649^{* * *}$ & $.406^{* * *}$ \\
\hline GT & 5.013 & 0.565 & & & & & 1 & $.363^{* * *}$ \\
\hline RO & 3.453 & 0.596 & & & & & & 1 \\
\hline
\end{tabular}

$* P \leq 0.1 ; * * P \leq 0.05 ; * * * P \leq 0.01$

Para evaluar la hipótesis, las variables independientes fueron introducidas una por una separadamente, comenzando con el control de variables (modelo 1), luego añadiendo solo EM (modelo 2), luego añadiendo solo GT (modelo 3) y finalmente, las dos variables independientes fueron introducidas juntas (modelo 4). Evaluamos la contribución de cada conjunto de variables determinando la importancia de la estadística-F asociada con el cambio en $\mathrm{R}^{2}$, después que cada conjunto fue introducido (Pedhazur and Schmelkin, 1991; Cagliano et al., 2006).

\section{VI.- RESULTADOS Y DISCUSIÓN}

Los resultados obtenidos usando el análisis de regresión jerárquica para el área de maquinaria se muestran en la Tabla 5. El modelo 1, el cual incluye solo variables contextuales, no muestran un efecto significativo y $\mathrm{H} 4$ no es apoyada. Cuando EM fue añadida a la regresión linear (modelo 2), los resultados presentaron un efecto significativo en RO mientras las variables contextuales no tuvieron efecto en ello $\left(\mathrm{R}^{2}=0.191, \mathrm{~F}=2.711, \mathrm{p}\right.$ 
$<0.05)$. En el modelo 3, GT mostro un efecto significativo mientras las variables contextuales no lo hicieron $\left(\mathrm{R}^{2}=0.254, \mathrm{~F}=3.916, \mathrm{p}<0.01\right)$.

Estos resultados apoyaron $\mathrm{H} 1$ y H2. Finalmente, ambos EM y GT fueron analizados juntos con las variables contextuales (modelo 4), en este caso GT mostraron el efecto más importante en RO $\left(\mathrm{R}^{2}=0.259, \mathrm{~F}=3.140, \mathrm{p}<0.05\right)$, mientras
EM fue menos importante. Las variables contextuales continúan siendo no significativas. Esto es porque EM y GT están correlacionadas, y consecuentemente la importancia de EM en el modelo 2 fue en realidad debido a su correlación con GT. El nivel más alto de la $\mathrm{R}^{2}$ fue alcanzado por el modelo 4. Esto significa que el modelo 4 predice RP mejor que en otros modelos. Este resultado del análisis apoya $\mathrm{H} 3$.

Tabla No. 5 - Variables contextuales, EM, GT y RO en el área de maquinaria

\begin{tabular}{|l|c|c|c|c|}
\cline { 2 - 5 } \multicolumn{1}{c|}{} & \multicolumn{4}{c|}{ RO } \\
\hline Factor & Modelo 1 & Modelo 2 & Modelo 3 & Modelo 4 \\
\hline $\begin{array}{l}\text { Capacidad productiva de } \\
\text { la fábrica }\end{array}$ & 0.112 & 0.132 & 0.077 & 0.088 \\
\hline Tamaño de la planta & -0.131 & -0.126 & -0.054 & -0.063 \\
\hline $\begin{array}{l}\text { Personalización del } \\
\text { producto }\end{array}$ & 0.231 & 0.086 & 0.094 & 0.071 \\
\hline EM & & $.333^{* *}$ & & 0.097 \\
\hline GT & & & $.429 * * *$ & $.368 * *$ \\
\hline & 1.734 & $2.711^{* *}$ & $3.916^{* * *}$ & $3.140^{* *}$ \\
\hline $\mathrm{F}$ & 0.1 & 0.191 & 0.254 & 0.259 \\
\hline $\mathrm{R}^{2}$ & 0.042 & 0.12 & 0.189 & 0.176 \\
\hline $\mathrm{R}^{2}$ ajustado & $* P \leq 0.1 ; * * P \leq 0.05 ; * * P \leq 0.01$ & & \\
\hline
\end{tabular}

En el área electrónica (Tabla 6), el modelo 1 muestra que el uso de la planta y el tamaño de la planta tiene una relación positiva y significativa con $\mathrm{RO}\left(\mathrm{R}^{2}=0.194, \mathrm{~F}=4.493, \mathrm{p}<0.01\right)$. Este resultado parcialmente apoya $\mathrm{H} 4$. Cuando EM fue añadido en el análisis (modelo 2), afecta significantemente el $\mathrm{RO}(\mathrm{p}<0.01)$, mientras que la capacidad productiva de la fábrica mantiene su relación positiva y significativa con el RO. Sin embargo, cuando GT fue añadido en el análisis (modelo 3), solo el uso de la planta y GT mostraron una relación positiva y significativa con el RO $(\mathrm{p}<0.01)$, mientras que el tamaño de la planta y la personalización del producto no lo hicieron. Este resultado apoya H1 y H2. Lo mismo fue cierto para todas las variables que fueron tomadas juntas (modelo 4) mientras que el efecto general fue un mejoramiento significante. De nuevo, esto es debido que EM y GT están correlacionadas, es por eso que en el modelo $2 \mathrm{EM}$ es significante. Este resultado apoya la H3. Esto provee cierto apoyo a la afirmación que GT debería de ser integrada con EM. 
Tabla No. 6 - Variables contextuales, EM, GT y RO en el área electrónica

\begin{tabular}{|l|c|c|c|c|}
\cline { 2 - 5 } \multicolumn{1}{c|}{} & \multicolumn{4}{c|}{ RO } \\
\hline Factor & Modelo 1 & Modelo 2 & Modelo 3 & Modelo 4 \\
\hline $\begin{array}{l}\text { Capacidad productiva de } \\
\text { la fábrica }\end{array}$ & $.337 * * *$ & $.352^{* * *}$ & $.367 * * *$ & $.366^{* * *}$ \\
\hline Tamaño de planta & $.348^{* * *}$ & $.235^{*}$ & 0.181 & 0.177 \\
\hline $\begin{array}{l}\text { Personalización del } \\
\text { producto }\end{array}$ & -0.077 & -0.128 & -0.092 & -0.105 \\
\hline EM & & $.362^{* * *}$ & & 0.111 \\
\hline GT & & & $.441 * * *$ & $.360^{* *}$ \\
\hline & $4.493 * * *$ & $6.183 * * *$ & $7.682 * * *$ & $6.174 * * *$ \\
\hline F & 0.194 & 0.31 & 0.358 & 0.364 \\
\hline $\mathrm{R}^{2}$ & 0.151 & 0.26 & 0.312 & 0.305 \\
\hline $\mathrm{R}^{2}$ ajustado & & & & \\
\hline
\end{tabular}

$* P \leq 0.1 ; * * P \leq 0.05 ; * * * P \leq 0.01$

\section{V.- CONCLUSIONES}

Esta investigación determinó variables contextuales que han tenido un efecto en la relación de GT, EM y RO en las áreas de maquinaria y electrónica. Los hallazgos muestran que GT afecta el RO en las áreas de maquinaria y electrónica. Además, la GT esta correlacionada con EM y esto provee cierto apoyo a la afirmación que GT debería estar integrada con EM.

Sin embargo, las variables contextuales analizadas no tuvieron una relación positiva con $\mathrm{RO}$ en el área de maquinaria y solo el uso de la planta en el área de electrónica muestra significancia. Investigaciones anteriores han confirmado la función del grupo SWL tamaño de la planta en la relación GT-RO. Existe un acuerdo general que el tamaño de la planta es una influencia valida en el efecto de GT en RO (Gilgeous, (2001); Beede and Young, (1998); Carlsson et al., (1994), Islam and Karim, 2011). Por lo tanto, este estudio apoya la relación del tamaño de la planta y GT en RO en el área electrónica.

En resumen, la evidencia sugiere que para ambas áreas la inclusión de GT mejora RO. Sin embargo, solo una de las variables contextuales ha tenido efecto en el área electrónica, es decir, el uso de la planta. Una causa posible de esta diferencia entre las áreas es el volumen de producción y de los costos de los activos y equipo usado en el proceso de manufactura. El área de maquinaria frecuentemente usa procesos laborales y compras por mayor que requieren equipos flexibles y de propósito general, que representan relativamente una producción de bajo volumen.

El área electrónica, por otro lado, produce normalmente grandes volúmenes de producción usando líneas equipadas con equipo altamente especializado. Esto representa una capacidad productiva de la fábrica alto, el cual es un factor importante para la difusión de depreciación en un gran número de unidades producidas, de este modo se reduce los costos por unidad. Esto también explica el efecto positivo de GT en RO en el área electrónica. En el caso de EM, su significancia desaparece con GT, probablemente porque EM se convierte en un calificativo para estas industrias.

Para los practicantes, esto genera un mayor interés en implementar prácticas de manufactura como ser EM y GT para competir en una economía globalizada. Además, los hallazgos de este estudio pueden tener alguna implicación práctica para las organizaciones de manufactura. Primero, las organizaciones de manufactura pueden mejorar el rendimiento de la planta por 
estableciendo e implementando las prácticas EM y GT. Segundo, las organizaciones de manufactura pueden mejorar el RO relacionando GT con EM y otras prácticas de manufactura para obtener ventajas competitivas. (Schroeder y Flynn, 2001). Tercero, los gerentes tienen que considerar la implementación de prácticas de GT para mejorar el RO.

Este estudio sufre de una limitación común relacionada con las opciones metodológicas. Un desafío mayor de investigación empírica es el problema de obtener muestras suficientes de datos válidos. Esto puede limitar las generalizaciones de los hallazgos investigativos a otros países e industrias.

Estudios futuros se enfocarán en evaluar EM, GT y variables contextuales para cada escala de RO separadamente para conocer que variable afecta directamente una medida operacional específica. Los análisis de variables contextuales también ayudan a futuros investigadores en alcanzar profundidad y relevancia practica en su investigación, pudiendo enfocarse en esas variables contextuales que son de importancia primordial. Además, este estudio puede extenderse a otras variables contextuales, PO e industrias para obtener su efecto en el RO. Asimismo, las relaciones entre EM y GT deben ser analizadas detalladamente, examinando su influencia positiva como mediadores o moderando variables en relación con el RO.
También incluye otras PO que pueden influenciar el mejoramiento de la relación de EM-RO que puede aportar nuevos aspectos para esta relación. Finalmente, sería interesante conducir un estudio longitudinal para determinar si las interrelaciones de las PO deben cambiar o evolucionar con cambios en el mercado. A este respecto, los proyectos con muchas iteraciones en diferentes años, como el proyecto HPM, construye una buena base para dicha investigación (Hallgren y Olhager, 2006).

\section{VI.- RECONOCIMIENTOS}

Este estudio ha sido conducido dentro de los marcos de los siguientes cuatro proyectos: (1) "PAR" del Instituto de Investigaciones Económicas y Sociales de la Universidad Nacional Autónoma de Honduras (UNAH); (2) "Acción especial” SGUIT (SBAPA-2015-06)-Junta de Andalucía (Proyecto 2015-148-US) "; (3) Junta de Andalucía (España) PAIDI Proyectos de Excelencia (P08-SEJ-03841); y (4) Programa Nacional de Diseño y Producción Industrial de España (DPI-2009-11148). Los autores desean expresar el reconocimiento a la UNAH, al Gobierno de Andalucía y al gobierno de España por su apoyo. Por otro lado, la presente investigación fue presentada en una versión anterior en inglés en el Congreso P\&OM 2016 y ganó el premio J. A. D. Machuca al mejor estudio del congreso. 


\section{REFERENCIAS}

Amahad, S., y Schroeder, R. (2002), "Refining the product-process-matrix", International Journal of Operations \& Production Management, Vol. 22, No. 1, pp. 103-124.

Ahmad, S., Schroeder, R., Sinha, K., (2003), "The role of infrastructure practices in the effectiveness of

JIT practices: implications for plant competitiveness", Journal of Engineering and Technology Management, Vol.20, pp. 161-191.

Amoako-Gyampah, K., y Acquaah, M. (2008), "Manufacturing strategy, competitive strategy and firm performance: An empirical study in a developing economy environment", International Journal Production Economics, Vol. 111, pp. 575-592.

Bates, K, Blackmon, K., Flynn, E.J. y Voss, C. (2001), "Manufacturing Strategy: Building Capability for Dynamic Markets". In R. G. Schroeder \& B. B. Flynn (Eds.), High Performance Manufacturing Global Perspectives (pp. 59-72). New York: John Wiley \& Sons, Inc.

Beaumont, N.B. y Schroder, R.M. (1997), "Technology, manufacturing performance and business performance amongst Australian manufacturers." Technovation 17, No. 6, pp. 297-307.

Beede, D. N. y Young, K. H., (1998), "Patterns of advanced technology adoption and manufacturing performance", Business Economics, Vol. 33, pp. 43-48.

Boyer, K.K., Ward, P.T. y Leong, K.G. (1996), "Approaches to the factory of the future: an empirical taxonomy", Journal of Operations Management, Vol. 14, pp. 297-313.

Boyer, K.K., Leong, K.G., Ward, P.T. y Krajewski, L.J. (1997), "Unlocking the potential of advanced manufacturing technologies", Journal of Operations Management, Vol. 15, No. 4, pp. 331-347.
Cagliano, R., y Spina, G. (2000), "Advanced manufacturing technologies and strategically flexible production." Journal of Operations Management 18, No. 2, pp. 169-190.

Cagliano, R., Caniato, F. y Spina, G. (2006), "The linkage between supply chain integration and manufacturing improvement programs", International Journal of Operations and Production Management, Vol. 26, $\mathrm{n}^{\mathrm{o}} 3$, pp. 282-299.

Corbett, L. M. (2008), "Manufacturing strategy, the business environment, and operations performance in small low-tech firms", International Journal of Production Research, Vol. 46, No. 20, pp. 5491-5513. Cronbach, L.J. (1951), "Coefficient alpha and the internal structure of tests", Psychometrika, Vol. 16, pp. 297-334.

Da Silveira, G. J.C. y Sousa, R. S. (2010), "Paradigms of choice in manufacturing strategy: Exploring performance relationships of fit, best practices, and capability-based approaches", International Journal of Operations \& Production Management, Vol. 30 No. 12, pp. 1219-1245.

Das, A., y Jayaram, J., (2003), "Relative importance of contingency variables for advanced manufacturing technology." International Journal of Production Research 41, No. 18, pp. 4429-4452.

Devaraj, S., Hollingworth, D., and Schroeder, R. G. (2004), "Generic manufacturing strategies and plant performance", Journal of Operations Management, Vol. 22, pp. 313-333.

Fine, C.H. (2000), "Clockspeed-based strategies for supply chain design", Production and Operations Management, Vol. 9, No.3, pp. 213-21.

Flynn, B.B., y Flynn, E.J. (1999), "Informationprocessing alternatives for coping with manufacturing environment complexity", Decision Sciences, Vol. 30, No. 4, pp. 1021-1052. 
Flynn, B.B., Sakakibara, S., y Schroeder, R.G. (1995), "Relationship between JIT and TQM: Practices and Performance", Academy of Management Journal, Vol. 38, No. 5, pp. 13251360.

Gilgeous, V. y Gilgeous, M. (2001), “A survey to assess the use of a framework for manufacturing excellence", Integrated Manufacturing Systems, Vol. 12 No. 1, pp. 48-58.

Groenevelt, H., (1993), The just-in-time system. In: Graves, S.C.(Ed.), Handbooks in Operations Research and Management Science. Elsevier, New York, NY, pp. 4.

Gyan-Baffour, G., (1994), “Advanced manufacturing technology, employee participation and economic performance: an empirical analysis", Journal of Managerial Issues, Vol. 6, pp. 491-505.

Hallgren, M., y Olhager, J. (2006), “Quantification in manufacturing strategy: A methodology and illustration", International Journal of Production Economics, Vol. 104, No. 1, pp. 113-124.

Hair, J.F. Jr, Black, W.C., Babin, B.J. y Anderson, R.J. (2011), Multivariate Analysis, PrenticeHall, Upper Saddle River, NJ.

Heine, M. L., Grover, V., y Malhotra, M. K. (2003), "The relationship between technology and performance: a meta-analysis of technology models", OMEGA, Vol. 31, pp. 189-204.

Im, J.H., and Lee, S.M., (1989), “Implementation of just-in-time systems in U.S. manufacturing firms", International Journal of Operations \& Production Management, Vol. 9, No1, pp. 5-14. Islam,

Mazharul and Karim, Azharul, (2011), "Manufacturing practices and performance: Comparison among small-medium and large industries", International Journal of Quality \& Reliability Management, Vol. 28 No. 1, pp. 43-61.

Ketokivi, M., y Schroeder, R.G. (2004), "Manufacturing practices, strategic fit and performance. A routine-based view", International Journal of Operations \& Production Management, Vol. 24, No. 2, pp. 171-191.

Koning, A. J. y Franses, P. H. (2003), “Confidence Intervals for Cronbach's Coefficient Alpha Values", ERIM Report Series Reference No. ERS-2003-041-MKT. Available at SSRN: http://ssrn.com/abstract $=423658$.

Lee, Wonhee; Rhee, Seung-Kyu y Oh Joongsan (2014), "The relationships between manufacturing strategy process, manufacturing-marketing integration, and plant performance: an empirical study of

Korean manufacturers", Operation Management Research, Vol. 7, pp. 117-133.

Machuca, J.A.D, Ortega-Jiménez, C.H., GarridoVega, P., y Ríos, José Luis Pérez Diez de los. (2011), "Do technology and manufacturing strategy links enhance operational performance? Empirical research in the auto supplier sector", International Journal of Production Economics, Vol. 133, No. 2, pp. 541-550.

Maier, F.H. y Schroeder, R.G. (2001), Competitive product and process technology. In R. G. Schroeder \& B. B. Flynn (Eds.), High Performance Manufacturing: Global Perspectives (pp. 96-116). New York: John Wiley \& Sons, Inc.

Malhotra M.K., Heine M.L., y Grover V. (2001), "An evaluation of the relationship between management practices and computer aided design technology", Journal of Operations Management; Vol.19, pp. 307-33.

Mallick, D. N. y Schroeder, R. G. (2005), “An Integrated Framework for Measuring Product Development Performance in High Technology Industries", Production and Operations Management, Vol. 14, No. 2, pp. 142-158.

Milling, P.M., Maier, F.H. y Mansury, D. (1999), Impact of manufacturing strategy on plant performance - insights from the international research project: world class manufacturing. 
Paper presented at the Managing Operations Networks EurOMA Conference, Venice, Italy, 573-580.

Morita, M. y Flynn, E. J. (1997), “The linkage among management systems, practices and behaviour in successful manufacturing strategy", International Journal of Operations \& Production Management, Vol. 17 No. 10, 1997, pp. 967-993.

Oltra, M.J. y Flor, M.L. (2010), “The moderating effect of business strategy on the relationship between operations strategy and firms' results", International Journal of Operations \& Production Management, Vol. 30, No. 6, pp. 612-638.

Pedhazur,E.y Schmelkin, L.(1991):Measurement, Design and Analysis: An Integrated Approach. Ed. Lawrence Erlbaum Associates, Hillsdale, NJ.

Papke-ShieldsKE,MalhotraMK,yGroveV(2006), "Evolution in the strategic manufacturing planning process of organizations", Journal of Operations Management, Vol. 24, pp. 421439.

Primrose, P.L., (1992), "Evaluating the introduction of JIT", International Journal of Production Economics, Vol. 27, pp. 9-22.

Raymond, L. (2005), “Operations management and advanced manufacturing technologies in SMEs: A contingency approach. Journal of Manufacturing Technology Management; Vol. 16, No. 7/8, pp.936955.

Rose, R.C., Kumar, N., y Ibrahim, H.I. (2008), "The Effect of Manufacturing Strategy on Organizational Performance", Performance Improvement, Vol. 47, No. 1, pp. 8-25.
Sakakibara, S., Flynn, B.B., Schroeder, R.G., y Morris, W.T. (1997), "The Impact of Just-InTime Manufacturing and its Infrastructure on Manufacturing Performance", Management Science, Vol. 43, No. 9, pp. 1246-1257.

Schroeder, R. G., y Flynn, B. (2001), High Performance Manufacturing-Global Perspectives. New York: John Wiley \& Sons, Inc.

Schroeder RG, Bates KA, y Junttila MA (2002), "A resource-based view of manufacturing strategy and the relationship to manufacturing performance", Strategic Management, Vol. 23, pp. 105-117.

Swamidass, P.M., and Kotha, S. (1998), "Explaining manufacturing technology use, firm size and performance using a multidimensional view of technology", Journal of Operations Management, Vol. 17, No.1, pp. 23-37.

Sun, H., and Gertsen, F., (1995), "Organizational changes related to advanced manufacturing technology in the production area", International Journal of Production Economics, Vol. 41, pp. 369-375. Thun, J.H. (2008), "Empirical analysis of manufacturing strategy implementation", International Journal of Production Economics, Vol. 113, pp. 370-382.

Tsai, K.H. (2004), “The impact of technological capability on firm performance in Taiwan's electronics industry", Journal of High Technology Management Research, Vol. 15, pp. 183-195. Van Bruggen, G.H., Lilien, G.L., and Kacker, M. (2002)." Informants in organizational marketing research: why use multiple informants and how to aggregate responses". Journal of Marketing Research, Vol. 39, No. 4, pp. 469-4 